\title{
REGISTROS ATRIBUIDOS A PLANTAS VASCULARES DEVÓNICAS EN LA PENÍNSULA IBÉRICA
}

\author{
Ángel MONTERO \\ Centro Paleobotánico. IMGEMA. Jardín Botánico de Córdoba. Avda. de Linneo \\ s/n. 14004 Córdoba.paleo.amontero@jardinbotanicodecordoba.com
}

Montero, A. 2008. Registros atribuidos a plantas vasculares devónicas en la Península Ibérica. [Reports of the Devonian vascular plants in the Iberian Peninsula.] Revista Española de Paleontología, 23 (2), 193-209. ISSN 0213-6937

\begin{abstract}
A critical analysis is presented of the sparse records in the literature of Devonian vascular plants in Spain and Portugal since the 19th Century. The first known reference is Hermite (1879). The records of specimens stored in Spanish and Portuguese Natural History museum collections are analysed, as well as their palaeobotanical value. The available information is summarised in two tables. All the specimens of Spanish Devonian vascular plants housed in Spanish museum collections are figured.
\end{abstract}

Keywords: Vascular plants, Devonian, Iberian Peninsula, palaeontological collections, museums.

\section{RESUMEN}

\begin{abstract}
Se revisan y valoran los descubrimientos publicados de flora vascular devónica encontrados en España y Portugal desde el siglo XIX, la primera cita conocida se debe a Hermite (1879), hasta la actualidad. También se analizan el origen y trayectoria de los ejemplares ibéricos de flora devónica que existen actualmente en instituciones españolas y portuguesas, así como su interés paleobotánico. Por último, se resume en dos tablas las publicaciones sobre flora devónica ibérica y los ejemplares de plantas vasculares devónicas depositados en museos españoles, y se figuran todos aquellos que permanecen en las colecciones de museos españoles.
\end{abstract}

Palabras clave: Flora vascular, Devónico, Península Ibérica, colecciones paleontológicas, museos.

\section{SUMMARY}

This paper reviews the scattered (and rather few) references to Devonian vascular plants in the Iberian Peninsula (Spain and Portugal). A critical analysis has been long overdue. The Devonian of Iberia is marine, and the remnants of vascular plants in marine strata are drifted remains, and generally quite fragmentary. Some of the records are indeed Devonian, but others involve Mississippian rather than Upper Devonian. In other cases ichnofossils may have been mistakenly recorded as plants. Where truly Devonian plants are involved as attested to by associated marine fossils the remains are highly fragmentary and difficult to identify properly.

The earliest quote is Hermite, 1879, but most are from the second half of the $20^{\text {th }}$ century.

Hermite (1879) records Archaeocalamites renaulti Hermite and Sphenophyllum maresi Hermite from Menorca in the Balearic Islands. The former was assigned to the
Lower Devonian, and the latter to the Middle Devonian. Although Devonian is present in Menorca, it is more than likely that the fossils reported by Hermite belong to the Mississippian, a subsystem which is widely represented in this island. The whereabouts of these unfigured specimens are unknown.

Gómez de Llarena (1950) recorded Psilophyton and Haliserites from the western Pyrenees in Navarra, and mentioned a Mid-Devonian age. In another paper, Gómez de Llarena (1956) figured a specimen identified as Psilophyton and mentioned Calamophyton, and remains resembling Halyserites and Asteroxylon from the same general locality. He also confirmed the Mid-Devonian age, based on marine faunal remains. Although the specimen figured on Psilophyton may be the same on that mentioned in the earlier paper, this is uncertain. The specimen figured as Psilophyton shows repeatedly dichotomised naked axes. It should be restudied, but the whereabouts of Gómez de Llarena's specimens are unknown. 
Nery Delgado (1908) mentioned fragmentary axes in few sites of the Barrancos Region (Alentejo, Portugal) and Teixeira (1951) figured and described Drepanophycus (aff. D. spinaeformis), Psilophyton? sp. and Hostimella? sp. (=Hostinella? sp.) from reputedly Lower Devonian strata at Barrancos. The specimens are very fragmentary and only some specimens show a single dichotomy. There is no apparent justification for the names employed. The whereabouts of the specimens cited and figured by Teixeira are currently unknown. The Early Devonian age is confirmed by palynology and fauna (Pereira et al, 1999; Oliveira et al., 2007), but some doubt remains since reworking of palynomorphs may have occurred.

Hartung (in Kullmann, 1960) identified Taeniocrada sp. from Lower to Middle Devonian strata in northern Palencia, Cantabrian Mountains (Palentian Domain). A later revision (Kullmann, pers. comm., 2008) suggests that these may have been ichnofossils.

From another locality in NE Palencia (Wagner, 1966) mentioned Protolepidodendron wahnbachense Kräusel \& Weyland, 1932, and Aneurophyton sp. These very fragmentary remains, which are figured in the present paper (Fig 2, $\mathrm{a}, \mathrm{b})$, are drifted plants found in association with goniatites, bivalves and ostracodes of early Famennian age (Weyer \& Blumenstengel, in Wagner et al., 1984: 17-19). The locality, near the village of Valle de Santullán, was originally assigned a Givetian age, which was corrected subsequently (op.cit.). Wagner (1971) redetermined P. wahnbachense as Hyenia sp. and the Aneurophyton sp. was redetermined by H. J. Schweitzer (in Wagner et al., 1984) as Sphenopteridium keilhauii Nathorst, 1902. The Hyenia sp. determination is questioned in the present paper but no alternative is proposed.This material is in the Palaeobotanical Collections of the Jardín Botánico de Córdoba (Córdoba, Spain).

Teixeira (1970) mentioned indeterminable plant remains of presumed Silurian age from Guadramil, near Bragança, Portugal. The whereabouts of these specimens are unknown, and the age was corrected subsequently (Teixeira \& Pais, 1973) as Late Devonian or Early Carboniferous.

In the 1970s several papers dealing with the Palaeozoic rocks of the province Zamora (Spain), near the Portuguese border recorded the presence of fossil plant remains in strata overlying a Silurian/Devonian sucession (Martínez García, 1972, 1973; Teixeira \& Pais, 1973). A locality at San Vitero (Zamora), sampled by E. Martínez García yielded masses of unidentifiable axial fragments among which a specimen of Eoacanthocarpus sp. was found together with two impressions of rootlet scars of Stigmaria ficoides Sternberg, 1825 (R. H. Wagner det.) The first taxon probably represents the fertile apex of an isoetalean lycopsid (compare Wagner, 2001: Fig. 24). Published records of this taxon are from the lower Mississippian, but its total range is unknown. Stigmaria ficoides seems exclusively Carboniferous. Teixeira \& Pais (1973) figured some poorly preserved remains from Alcañices (Zamora) as cf. Calamites sp. and cf. Sublepidodendron sp., giving the age as either Late Devonian or Early Carboniferous. Although the evidence is weak, all the plant fragments from Alcañices and the neighbouring area of Guadramil (Bragança, Portugal), may refer to the same stratigraphic interval of either Late Devonian or Early Mississippian age. The whereabouts of these specimens are unknown.

A similar deposit of drifted plant remains was found in the province Badajoz in SW Spain, not far from the Portuguese border. A long list of taxa identified by Álvarez Ramis (1981), was accompanied by illustrations of axial fragments which recalled similar assemblages at Barrancos (Alentejo, Portugal). The published list is a mixture of Devonian and Mississippian taxa, unlikely to be found in a single locality. The illustrations show this material to be indeterminable. Originally assigned an Later Devonian age by Álvarez Ramis, (1981), this opinion was later corrected to either Late Devonian or Early Carboniferous (Álvarez Ramis in Herranz Araujo, 1985: 793).

Another paper by Álvarez Ramis (1988) mentions the presence of Sciadophyton steinmanni Kräusel \& Weyland, 1930, in a different locality of the province Badajoz (Spain), to which a Gedinnian age was attributed. The specimen in question was neither illustrated nor described, and its whereabouts are unknown.

Finally, Pardo Alonso (1997) mentioned the presence of Lepidodendraceae (=Flemingitaceae) (Fig. 2.c) among a mass of unidentifiable plant fragments from an horizon in between upper Emsian and upper Givetian at Guadalmez (Ciudad Real province, Spain). The overlying Frasnian deposits are well dated (op. cit.). This material is in the Palaeontological Collections of the Geology Department, University of València (Spain).

Apart from these published records, there are a few possible Devonian remains to which the present writer has had access.

Two poorly preserved lycopsid axes from Cangas de Onís in the Asturias (Spain) are figured here (Fig. 2.d-e). These remains, collected by M. Julivert, from the Ermita Sandstone Formation of Late Famennian to Early Tournaisian age, are in the Palaeobotanical Collections of the Jardin Botánico de Córdoba.

A specimen of Archaeocalamites from the province of Huesca (Pyrenees, Spain) is also figured here (Fig. 2.f). This specimen belongs to the Museo Geominero, Madrid. Although locality details are unavailable, it is attributed to the Upper Devonian. However, Archaeocalamites is commonly recorded from the Mississippian.

A single specimen from the Lower Devonian of Barcelona and which is in the Museo Geológico del Seminario de Barcelona, may not be a plant remain at all (Fig. 2.g).

The various repositories of putative Devonian land plants in Spain and Portugal are listed in Table 2. The Devonian remains not figured in previous papers and lodged in Spanish museums, are illustrated in figure 2. 


\section{INTRODUCCIÓN Y ANTECEDENTES}

El Devónico es un periodo de extrema importancia desde un punto de vista paleobotánico ya que en el se produce la gran diversificación de las plantas terrestres.

Los hallazgos más importantes corresponden al Devónico Inferior de Rhynie Chert (Escocia). La flora de esta localidad ha sido estudiada minuciosamente (Remy et al., 1994; Edwards et al., 1998; Roth-Nebelsick et al., 2000; Kerp et al., 2001; Taylor \& Krings, 2005; Taylor et al., 2005,2006 ) y hasta la fecha han sido descritas diversas traqueofitas de los órdenes Rhyniales, Trimerophytales, Zosterophyllales y Drepanophycales acompañadas por hongos, líquenes, cianobacterias, nematofitos y algas.

Durante el Devónico Medio aparecen los hábitos arbustivo y arborescente (Stein et al., 2007) y se produce la desaparición de la mayoría de los grupos dominantes en el Devónico Inferior, excepto las Lycopsida, y la aparición de nuevos grupos, como el de las Progymnospermopsida (Rhacophytales) y otros como las Coenopteridales (Pteropsida) y las Cladoxylales, grupo problemático incluido en las Pteropsida (Cleal, 1993). Será en el Devónico Superior donde, además de vegetación arbustiva, proliferen los árboles de porte considerable. Un yacimiento clásico del Devónico Medio es el de Goé, en La Vesdre (Bélgica) con abundantes Cladoxylales, entre las que destaca Pseudosporochnus Potonié \& Bernard, 1904 (Berry \& Fairon-Demaret, 1997).

Tras la extinción del límite Frasniense-Fameniense aparecen las Pteridospermopsida y las Sphenopsida, dentro de las Lycopsida, las Lycopodiales, y el género Archaeopteris Dawson, 1861 (Progymnospermopsida) aumenta su presencia de manera exponencial formando grandes extensiones de bosques. Un yacimiento clásico del Devónico Superior es el de Kilkenny en Kiltorcan Hill (Irlanda), con gran abundancia de la licopodial arborescente Cyclostigma Haughton, 1859, y de la progimnosperma Archaeopteris (Jarvis, 2000).

Al final del Devónico Superior, se encuentran la mayoría de los grandes grupos botánicos presentes en la actualidad, con excepción de las Coniferopsida y Cycadopsida, que aparecen en el Carbonífero, y las angiospermas y Gnetopsida que se registran a partir del Mesozoico.

Si se habla de la explosión cámbrica como el "momento" de aumento y diversificación de vida en los mares, hay que hablar de la gran explosión devónica como el "momento", no de la "invasión" pero sí de la total colonización de las áreas continentales por parte de los vegetales, y de su diversificación.

Actualmente los estudios sobre floras devónicas han experimentado un gran auge como lo demuestran los trabajos sobre floras de China, Antártida, Ártico, Estados Unidos, Inglaterra, Marruecos, Argentina, Brasil, Venezuela, entre otros (Guerrienne et al., 2001, 2006; Edwards et al., 2001; Kerp et al., 2001; Meyer-Berthaud \& Gerrienne, 2001; Hao et al., 2004; Soria \& Meyer-Berthaud, 2004; Berry,
2005; Hammond \& Berry, 2005; Wang \& Xu, 2005; Wang et al., 2005a, 2005b; Gensel \& Kasper, 2005; Cressler \& Pfefferkorn, 2005; Berry \& Wang, 2006; Edwards, 2006; Wang \& Berry, 2006; Gensel \& Albright, 2006; Taylor et al., 2006; Xu \& Berry, 2008)

Por el contrario, el número de estudios sobre floras devónicas en la Península ibérica es exiguo, y limitado a hallazgos puntuales de restos vegetales flotados que fosilizaron en ambiente marino y han sido colectados en su mayoría en afloramientos correspondientes a borde de cuenca (Hermite, 1879; Gómez de Llarena, 1950; Teixeira, 1951, 1970; Kullmann, 1960; Wagner, 1966, 1971, 1984; Martínez García, 1972, 1973; Teixeira \& Pais, 1973; Álvarez-Ramis, 1981, 1988; Pardo Alonso, 1997). Esta carencia puede ser debida al desconocimiento o a la inexistencia en la Península de afloramientos de origen continental de esta edad

Además de los escasos fósiles de plantas vasculares que se encuentran actualmente en instituciones españolas, también veremos los antecedentes bibliográficos que la literatura paleontológica nos ha dejado y que indican el descubrimiento esporádico pero continuo de plantas vasculares terrestres en estratos devónicos en la Península, la mayoría difícilmente contrastables.

\section{REGISTROS DE FLORA VASCULAR EN EL DEVÓNICO DE LA PENÍNSULA IBÉRICA}

\section{CUENCA BALEAR: MENORCA (ISLAS BALEA- RES, ESPAÑA)}

La referencia más antigua que conocemos es la de Hermite (1879), quién en un trabajo sobre las Islas Baleares, cita en Menorca dos especies de plantas que asigna al Devónico. La primera es el Archaeocalamites renaulti Hermite, 1879, encontrado en varios lugares: en la rada de Mahón en unas pizarras azuladas, también en la alquería de Terra Rotje en una intercalación de pizarras y areniscas pardo-amarillentas y, por último, en Rafal Rotje, cerca de Mercadal, en una intercalación de areniscas grises y pizarras negras. La segunda es el Sphenophyllum maresi Hermite, 1879, que se encuentra junto con la especie anterior en las mismas pizarras azuladas de la rada de Mahón. También menciona haber encontrado "vestigios de plantas" en Mercadal y "jarillas de plantas" cerca de esa localidad, así como "vegetales mal conservados" en Rafal Rotje, "muchas impresiones vegetales" en la alquería de Santa Rita y "jarillas vegetales" en la Masía de San Isidoro, todo ello de supuesta edad devónica (ver Tabla 1). La flora citada, pero no figurada, por Hermite está recogida por Mallada (1892) en su "Catálogo General de las especies fósiles encontradas en España", aunque asignando el género Archaeocalamites Stur, 1875, al Devónico Inferior y Sphenophyllum Brongniart, 1828, al Devónico Medio, y 


\begin{tabular}{|c|c|c|c|c|}
\hline $\begin{array}{l}\text { Autor y año de la } \\
\text { publicación }\end{array}$ & Especie & $\begin{array}{l}\text { Edad asignada en } \\
\text { la cita original }\end{array}$ & $\begin{array}{l}\text { Edad asignada } \\
\text { con posterioridad }\end{array}$ & Localidad \\
\hline Hermite (1879) & $\begin{array}{l}\text { Archaeocalamites renaulti Hermite, } 1879 \\
\text { Sphenophyllum maresi Hermite, } 1879\end{array}$ & Devónico & & $\begin{array}{l}\text { Terra Rotje, Rafal Rotje, Mercadal, } \\
\text { Rada de Mahón (Menorca). } \\
\text { Rada de Mahón (Menorca) }\end{array}$ \\
\hline Gómez de Llarena (1950) & $\begin{array}{l}\text { Psilophyton } \mathrm{sp} . \\
\text { Haliserites } \mathrm{sp} .\end{array}$ & Devónico Med. & & Quinto Real (Navarra). \\
\hline Teixeira (1951) & $\begin{array}{l}\text { Drepanophycus sp. (aff. D. spinaeformis) } \\
\text { Psilophyton? sp. } \\
\text { Hostimella? sp. }\end{array}$ & Devónico & Devónico Inf. & Barrancos (Alentejo, Portugal) \\
\hline Gómez de Llarena (1956) & $\begin{array}{l}\text { Psilophyton } \mathrm{sp} . \\
\text { Haliserites } \mathrm{sp} . \\
\text { Calamophyton } \mathrm{sp} .\end{array}$ & Devónico Med. & & $\begin{array}{l}\text { Quinto Real (Navarra) } \\
\text { Olaberri (Navarra) }\end{array}$ \\
\hline Kullmann (1960) & Taeniocrada sp. & $\begin{array}{l}\text { Siegeniense- } \\
\text { Eifeliense }\end{array}$ & & Valle del Arruz (Palencia) \\
\hline Wagner (1966) & $\begin{array}{l}\text { Protolepidodendron wahnbachense Kräusel \& } \\
\text { Weyland } \\
\text { Aneurophyton } \mathrm{sp} .\end{array}$ & Devónico Med. & Fameniense inf. & Valle de Santullán (Palencia) \\
\hline Wagner (1971) & $\begin{array}{l}\text { Hyenia sp. } \\
\text { Aneurophyton sp. }\end{array}$ & ¿Givetiense? & Fameniense inf. & Valle de Santullán (Palencia) \\
\hline Teixeira \& Pais (1973) & $\begin{array}{l}\text { cf. Calamites sp. } \\
\text { cf. Sublepidodendron sp. }\end{array}$ & $\begin{array}{l}\text { Devónico Sup.- } \\
\text { Carbonífero Inf. }\end{array}$ & & Alcañices (Zamora) \\
\hline Wagner (1984) & $\begin{array}{l}\text { Hyenia sp. } \\
\text { Sphenopteridium keilhauii Nathorst }\end{array}$ & Fameniense inf. & Fameniense inf. & Valle de Santullán (Palencia) \\
\hline Álvarez-Ramis (1981) & 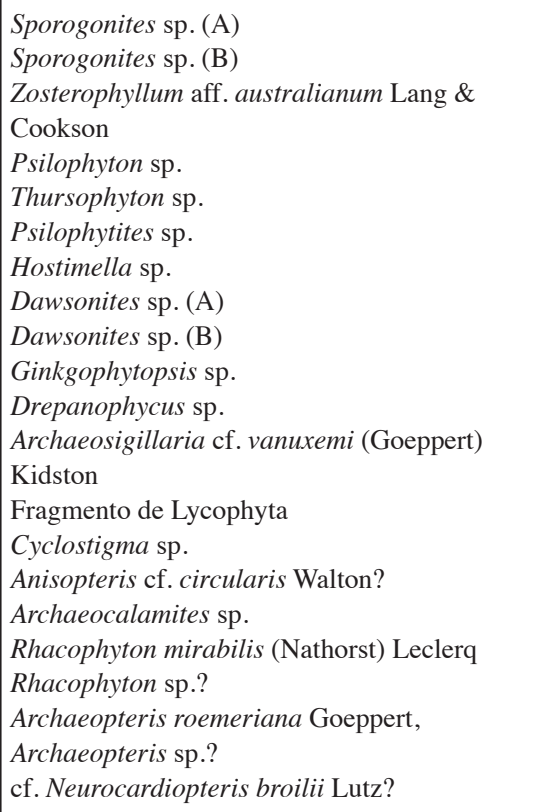 & Devónico Sup. & $\begin{array}{l}\text { Devónico Sup.- } \\
\text { Carbonífero Inf. }\end{array}$ & $\begin{array}{l}3 \mathrm{~km} \text { al SE de Sierra de Hornachos } \\
\text { (Badajoz) }\end{array}$ \\
\hline Álvarez-Ramis (1988) & Sciadophyton steinmanni Kräusel \& Weyland & Gediniense & & Monterrubio de la Serena (Badajoz) \\
\hline Pardo Alonso (1997) & Lepidodendraceae indet. & $\begin{array}{l}\text { Emsiense sup. } \\
\text {-Givetiense sup. }\end{array}$ & & Guadalmez (Ciudad Real) \\
\hline
\end{tabular}

Tabla 1. Especies citadas de flora vascular devónica encontradas en la Península Ibérica. No figuran las publicaciones en las que no se citan taxones de plantas vasculares y tampoco se reflejan las localidades con material fragmentario sin identificar (Ver texto). Species cited or figured of Devonian vascular plants reported from the Iberian Peninsula (Only where the taxa were identified, either correctly or incorrectly - see text). 
con una errata en la fecha de publicación de la traducción del trabajo de Hermite en castellano. Si bien las dataciones de Hermite de yacimientos con fauna marina parecen ser acertadas, en el caso de los yacimientos con restos vegetales fósiles son de dudosa fiabilidad.

Las dos especies citadas por Hermite son claramente carboníferas. El propio Mallada (1898), inicialmente sólo parece considerar en el Devónico español el registro de Archaeocalamites renaulti, afirmando categóricamente que "es el único resto del reino vegetal de que hasta la fecha se tenga noticia", aunque en páginas posteriores también cita Sphenophyllum maresi y repite las localidades dadas por Hermite. En el Mapa Geológico, escala 1:200.000 (Bourrouilh, 1970-1972), la parte norte de la rada de Mahón se asigna al Carbonífero Inferior de "facies Culm" (intercalaciones de materiales terrígenos en capas marinas) y aunque aparecen manchas de Devónico en el centro de la isla cerca de Mercadal, lo que ocupa grandes extensiones en dicha zona es también el "Culm". En la Memoria explicativa del Mapa se citan varias especies vegetales encontradas al este de la isla, todas ellas carboníferas, y se menciona haber encontrado "en numerosos puntos" Asterocalamites $\mathrm{sp}$. (=Archaeocalamites $\mathrm{sp}$.), pero siempre en el Carbonífero Inferior.

El caso del género Archaeocalamites merece un comentario aparte, porque es un género común en el Carbonífero Inferior, pero existen algunas referencias en las que se cita este género en estratos devónicos, como hemos visto en la publicación de Hermite.

Stur (1875) creó este género con material procedente del Carbonífero Inferior de Alemania, Stockmans (1940) dejó claro que era bastante cuestionable que este género pudiera aparecer por debajo del Carbonífero; Boureau (1964) describe varias especies todas del Carbonífero Inferior y Wagner $(1984,2001)$ lo sitúa exclusivamente en el Misisípico (Tournaisiense, Viseense y Namuriense inferior o Serpujoviense en la escala rusa). Gensel \& Andrews (1984) comentan que algunas impresiones de troncos recolectadas en yacimientos devónicos de Bear Island (Spitsbergen) y Alemania han sido asignadas a este género, pero que no deja de ser dudosa la asignación dada la pobreza del material. Cleal (1993) cita una referencia bibliográfica que sitúa Archaeocalamites en el Fameniense, aunque no le da mucho crédito cuando en las mismas páginas considera que la Familia tiene un alcance estratigráfico que va desde el Ivoriense (Tournaisiense superior) al Asbiense (Viseense superior). Lo mismo ocurre con otra mención que sitúa este género [en concreto ?Archaeocalamites radiatus (Brongniart, 1828) Stur, 1875] en el yacimiento devónico de Plaistow Quarry (Devon, Inglaterra) y que también es cuestionada por los autores que lo citan (Cleal \& Thomas, 1995). Mamay \& Bateman (1991) y, posteriormente, Berry \& Fairon-Demaret (2001) consideran dudosa su presencia por debajo del Tournaisiense. Hay que añadir que aunque es fácil la identificación de los contramoldes internos del tallo, ya que tienen los caracteres diagnósticos muy claros (costillas no alternantes en los nudos), si la preservación no es buena, máxime cuando se trata de material flotado, puede haber confusiones en la identificación. Por otro lado, en más de un caso habría que cuestionar la asignación estratigráfica.

\section{PIRINEOS: NAVARRA (ESPAÑA)}

La segunda cita es tan sólo un párrafo dentro de una nota breve de Joaquín Gómez de Llarena (1950), en donde describe haber encontrado "restos escasos y poco completos de Psilophyton y Haliserites en unas pizarras grises" cerca de la localidad de Quinto Real, en el Pirineo navarro, que atribuye al Devónico Medio por su posición estratigráfica. No hay figuración y la escueta descripción del autor lleva a pensar que los restos consistirían en fragmentos de tallos estériles y briznas maceradas. Sin embargo, en un trabajo posterior sobre esta flora (Gómez de Llarena, 1956), sitúa estos mismos hallazgos en una potente formación de pizarras negras, bastas y deleznables, que se continúan desde Quinto Real hasta Olaberri (Navarra). Las atribuciones taxonómicas son prudentes, mencionando impresiones de Psilophyton Dawson, 1859 o similares a Asteroxylon Kindston \& Lang, 1920, otras del tipo Haliserites Sternberg, 1833, y otras referibles a Calamophyton Kräusel \& Weyland, 1926. Confirma la edad probable de Devónico Medio para el tramo entre Urquiaga y Olaberri basándose en el descubrimiento de un ejemplar del cnidario Calceola sandalina. En este trabajo sí figura un ejemplar de Psilophyton, que parece presentar tres e incluso cuatro dicotomías de una parte vegetativa. Se desconoce el paradero de todo este material a pesar de haberse realizado gestiones para su localización (Begoña Sánchez, 2005, com. escrita).

La descripción de las partes estériles del género Psilophyton Dawson, 1859, ha sido tan poco determinativa que ha servido muchas veces para poder incluir dentro de este taxón restos fragmentarios de difícil determinación. Así, Hartman \& Banks (1980) afirman que las especies del género Psilophyton dadas entre 1871 y 1968 necesitan ser revisadas porque la descripción del género ha sido refinada, una de las especies originales: Psilophyton princeps var. ornatum Dawson, 1871, ha sido transferida al género Sawdonia Dawson, 1871 por Hueber (1971), y se han definido nuevas especies en estas últimas décadas.

El género Haliserites Sternberg, 1833, fue establecido en el Cenomaniense de Sajonia (Alemania) con la especie $H$. reichii. En 1847 y 1852, Göppert usó el término genérico para $H$. dechenianus del Devónico Inferior, siendo posteriormente utilizado para designar diversos restos fragmentarios y/o mal preservados del Devónico, hasta que en 1903 White expuso la imposibilidad de la utilización de un nombre genérico de esta forma e introdujo el nuevo nombre de Taeniocrada (ver Höeg, 1967). 


\section{ZONA CENTROIBÉRICA: BAIXO ALENTEJO (PORTUGAL)}

La primera noticia sobre la presencia de flora devónica en Portugal es la de Nery Delgado (1908: 175, 209), quién encuentra en Monte das Eiras Altas, Volta dos Nogais y en Noudar (Barrancos, Alentejo) restos fragmentarios de caules que no determina ni figura y que considera de la parte alta del Gotlandiano (Silúrico) o del Devónico. Este material se encuentra actualmente en las colecciones del Museu Geológico del Instituto Nacional de Engenharia, Tecnologia e Inovação (Miguel Ramalho, 2008, com. escrita).

En 1950 Teixeira visita en Barrancos las localidades con flora estudiadas por Nery Delgado (1908), y en 1951 publica un trabajo sobre una flora encontrada por él en tres lugares de esta región. Los fósiles se encuentran en los niveles de lutitas y grauvacas que componen la Formación Terena (Barrancos). Basándose en la flora encontrada, Teixeira en este trabajo (1951) y en otros posteriores (1982a, 1982b) asigna estas capas al Devónico Inferior y considera que se han formado en un ambiente litoral de poca profundidad. Aunque Teixeira (1951) había citado el trabajo de Nery Delgado (1908), Teixeira \& Thadeu (1967) citan el trabajo del primero (Teixeira, 1951) como el único descubrimiento de plantas vasculares devónicas en Portugal, hasta que en 1970 el mismo Teixeira publica la flora de Guadramil (Bragança).

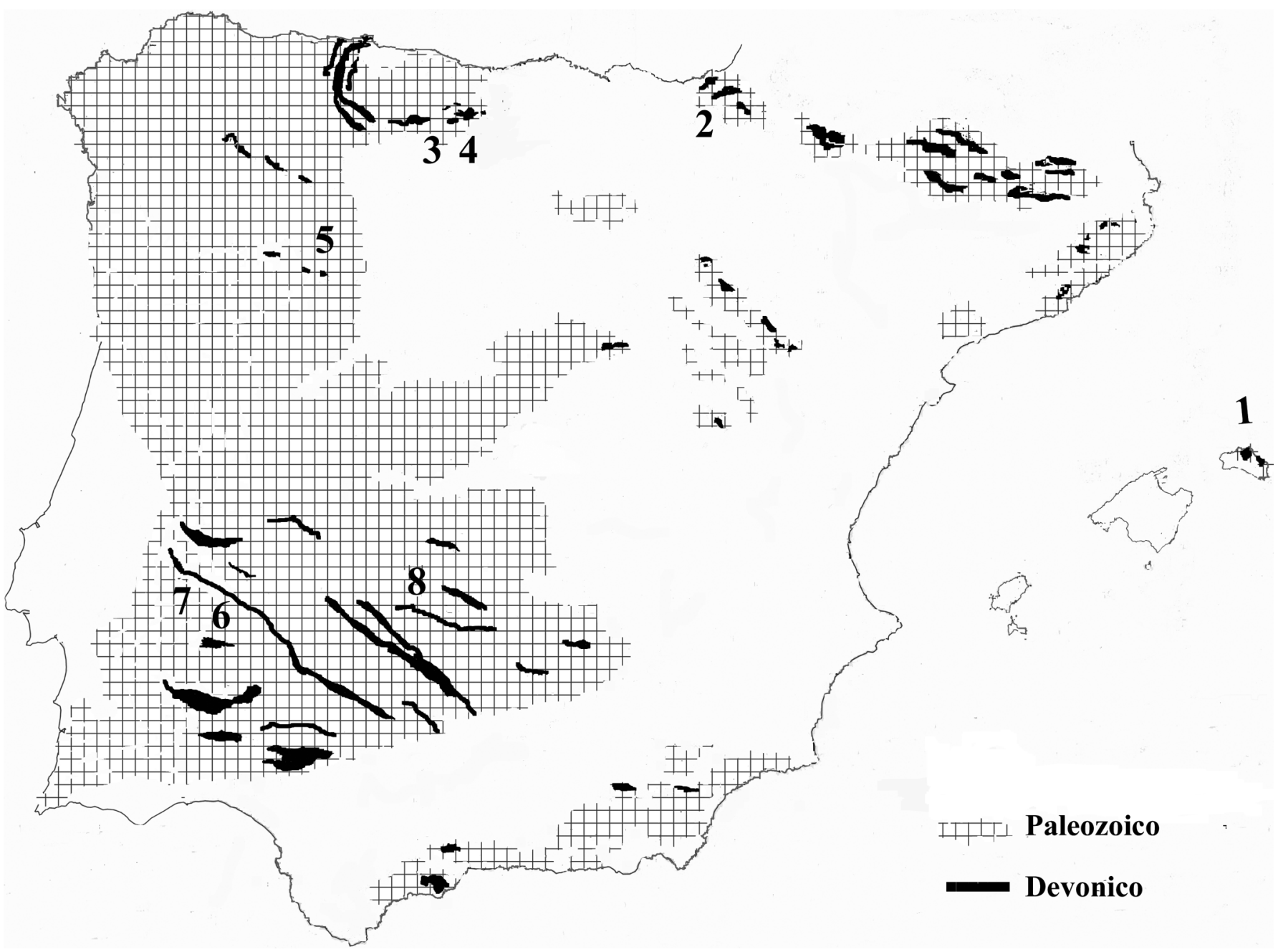

Figura 1. Distribución de afloramientos devónicos en la Península Ibérica con la situación de los hallazgos de plantas devónicas citados en la literatura y comentados en este trabajo. Sólo se señalan las citas en las que se determinaron, correcta o incorrectamente, los taxones encontrados (ver texto). 1, Menorca (Hermite, 1878); 2, Pirineo navarro (Gómez de Llarena, 1950); 3, N de Palencia (Kullmann, 1960); 4, NE de Palencia (Wagner, 1966); 5, Zamora (Teixeira \& Pais, 1973); 6, Badajoz (Álvarez-Ramis, 1981, 1982); 7, Barrancos (Teixeira, 1951); 8, Ciudad Real (Pardo, 1997).

Distribution of Devonian outcrops in the Iberian Peninsula showning the position of plant fossil localities as reported in the literature, and discursed in the present paper (only where the Devonian plant fossils were determined, correctly or incorrectly - See text). 1, Menorca (Hermite, 1878); 2, Navarrese Pyrenees (Gómez de Llarena, 1950); 3, Northern Palencia (Kullmann, 1960); 4, NE Palencia (Wagner, 1966); 5, Zamora (Teixeira \& Pais, 1973); 6, Badajoz (Álvarez-Ramis, 1981, 1982); 7, Barrancos (Teixeira, 1951); 8, Ciudad Real (Pardo, 1997). 
Teixeira (1951) cita impresiones de restos de caules "de tipo" Drepanophycus Göppert, 1952, con semejanzas a D. spinaeformis Göppert, 1952. Otros fragmentos de caules lisos más estrechos y dicótomos los asigna con dudas a Psilophyton y Hostimella (=Hostinella). El material, al igual que ocurre en el trabajo de Álvarez-Ramis (1981), que veremos posteriormente, es bastante deficiente tal como da a entender Teixeira en el texto y se observa en las figuras, además las asignaciones taxonómicas las realiza con muchas precauciones. Trabajos posteriores han confirmado la edad devónica para esas capas basándose en graptolitos y miosporas (Pereira, et al., 1999; Oliveira et al., 2007), aunque puede haber habido retrabajamiento de las esporas, dado los taxones implicados.

Los ejemplares se encontraban en la colección de los antiguos Serviços Geológicos de Portugal (com. personal Roberto Wagner, 2007), hoy Museu Geológico del Instituto Nacional de Engenharia, Tecnologia e Inovação, pero actualmente este material no se encuentra en dicha Institución (Miguel Ramalho, 2008, com. escrita).

Por último, en 1964, J. Perdigão colecta restos fragmentarios de vegetales devónicos en Santa Iria (Serta), que no publica, y permanecen sin determinar en el Museu Geológico del Instituto Nacional de Engenharia, Tecnologia e Inovação (Miguel Ramalho, 2008, com. escrita).

\section{ZONA CANTÁBRICA: PALENCIA (ESPAÑA)}

Una cuarta cita de flora vascular en España aparece, de manera tangencial, en una memoria sobre ammonoideos devónicos de la Cordillera Cantábrica (Kullmann, 1960). Los ejemplares fueron colectados en las denominadas por Kullmann "Capas de Arruz" (Arroyo de Arauz, Valle del Arauz, NO Palencia), en niveles de margas y margas nodulosas con briznas vegetales. Estas capas fueron datadas por la presencia de goniatítidos, con un lapso de edad que iba desde el Siegeniense al Eifeliense. Las "Capas de Arruz" equivalen al Miembro Requejada y a una pequeña parte de la sucesión suprayacente dentro de la Formación Abadía (García-Alcalde et al., 1988).

Entre el material colectado W. Hartung, en comunicación escrita a Kullmann (Kullmann, 1960: 467), menciona la presencia de Taeniocrada sp., pero el ejemplar no se figura en la publicación. Otros autores, en una revisión posterior sin publicar, sugirieron que podían ser en realidad icnofósiles (pistas vermiculares; Jürgen Kullmann, 2008, com. escrita). Todo este material fue depositado en el Geologisch-Paläontologischen Institut, Universität Tübingen (Alemania), pero Kullmann desconocía su paradero (Jürgen Kullmann, 2008, com. escrita).

En el hipotético caso de que realmente fuera un resto vegetal, podrían no ser restos flotados si, como parece, este género fuera subacuático (Schweitzer, 1990). Hartung reconocía que el material era bastante deficiente y no podía llegar a concretar la especie. Muchas atribuciones a Taeniocrada White, 1903, resultan dudosas porque han sido asignados a este género caules sin estructuras anatómicas determinativas salvo una única estría longitudinal en el caule, acabando convertido el género en un auténtico "cajón de sastre" (Brauer, 1981; Gensel \& Andrews, 1984: 340).

Por todo lo dicho anteriormente la determinación de estos ejemplares dada por Hartung debe ser puesta en duda.

Las siguientes citas se refieren a material colectado, cerca del pueblo de Valle de Santullán (NE de Palencia) en 1953, (localidad 316 del Dr. Wagner), en el Miembro Revilla de la Formación Vidrieros, en capas con abundante fauna de bivalvos y escasos ostrácodos, trilobites y goniatítidos. Fue datado con bivalvos, fragmentos de goniatítidos y restos de plantas vasculares como Devónico Medio, probable Givetiense (Wagner, 1966). Estudios posteriores de la fauna de un yacimiento en una localidad cercana a Valle de Santullán permitían situar estratigráficamente aquel yacimiento en el Fameniense inferior y la especie de goniatítido que había permitido asignarlo a una edad Givetiense fue reestudiada, considerándose la determinación anterior errónea (Wagner et al., 1984). Por tanto, el yacimiento quedó definitivamente asignado al Fameniense inferior, por lo que, en lo que a la flora se refiere, quedaría incluido en la Biozona VI, de Archaeopteris, de Banks (1981).

El escaso material vegetal colectado consiste en tres muestras de mano con dos especies, una de ellas presenta molde y contramolde. Los resultados del estudio del material fueron publicados por Wagner (1966) asignando uno de los ejemplares al taxón Aneurophyton sp. (Fig. 2.a) (Progymnospermopsida, Aneurophytales) y el otro a Protolepidodendron wahnbachense Kräusel \& Weyland, 1932 (Lycopsida, Protolepidodendrales) (Fig. 2.b), que FaironDemaret (1979) en un cambio nomenclatural denomina Estinnophyton wahnbachense (Kräusel \& Weyland, 1932) Fairon-Demaret, 1979. Posteriormente el ejemplar determinado como Protolepidodendron wahnbachense Kräusel \& Weyland, 1932, del que se conserva huella y contrahuella, fue redeterminado (Wagner, 1971) como Hyenia sp. (Pteropsida, Cladoxylales).

En cuanto al ejemplar identificado como Aneurophyton sp. fue redeterminado por H. J. Schweitzer (Wagner et al., 1984) como Sphenopteridium keilhauii Nathorst, 1902, especie definida en el Devónico Superior alto de Bear Island (Spitsbergen).

\section{ZONA CENTROIBÉRICA: BRAGANÇA(PORTUGAL)}

En 1970 Teixeira descubre en Guadramil (Bragança, Portugal), en unas rocas de aspecto grauváquico, unos restos escasos y muy fragmentarios de flora vascular, se trata de pequeños fragmentos de caules que el autor considera inclasificables y que asigna al Silúrico (Teixeira,1970). Sólo figura un ejemplar que parece un pequeño resto de caule sin ninguna dicotomía. En una publicación con Pais (Teixeira \& Pais, 1973), que veremos más adelante, se asigna este material al Devónico Superior o Carbonífero Inferior. 


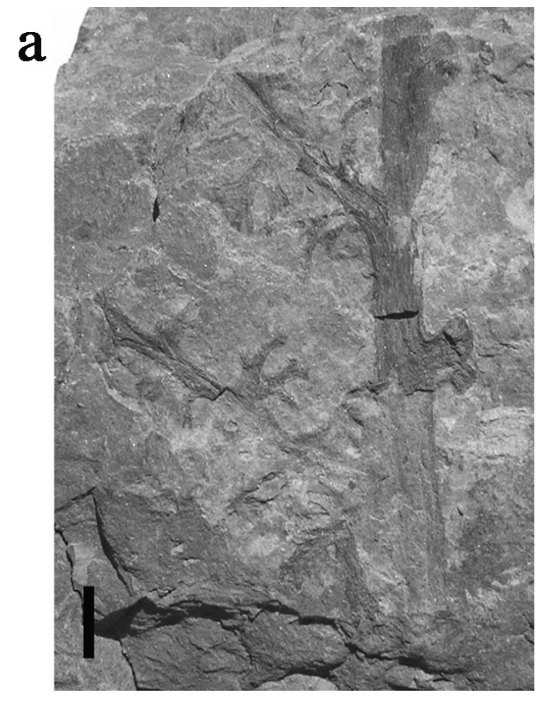

b

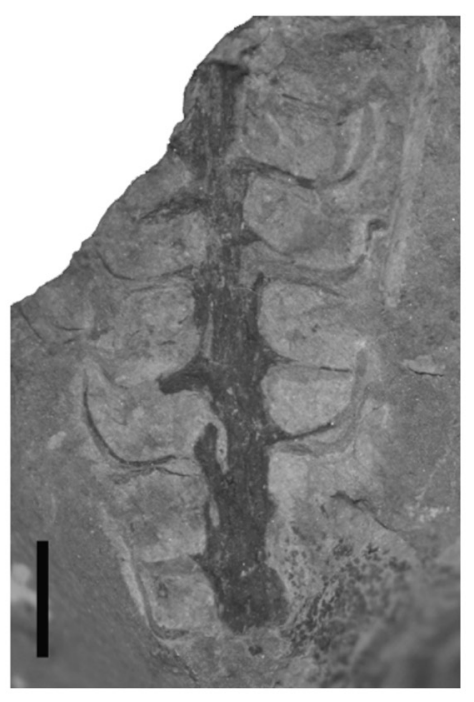

d

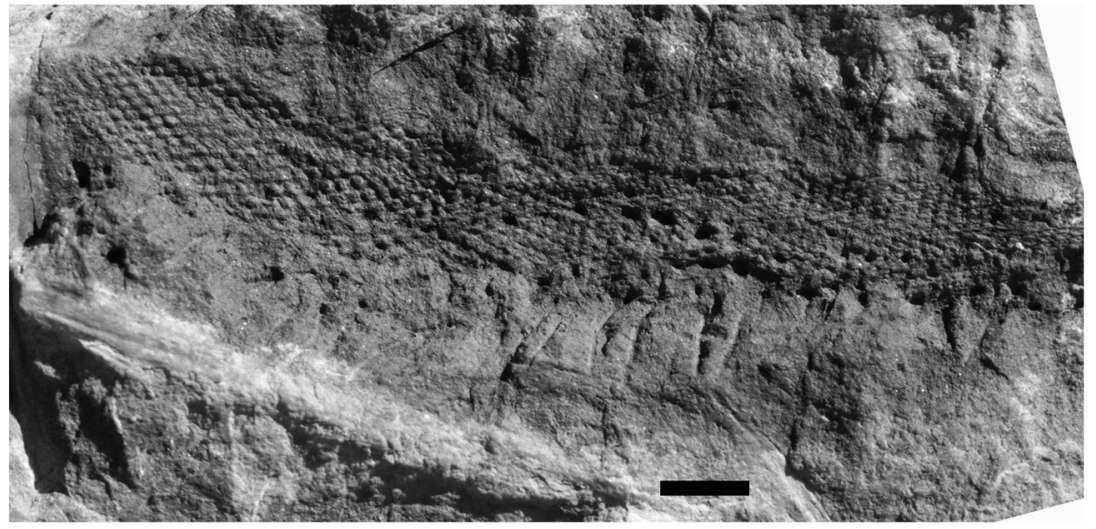

e

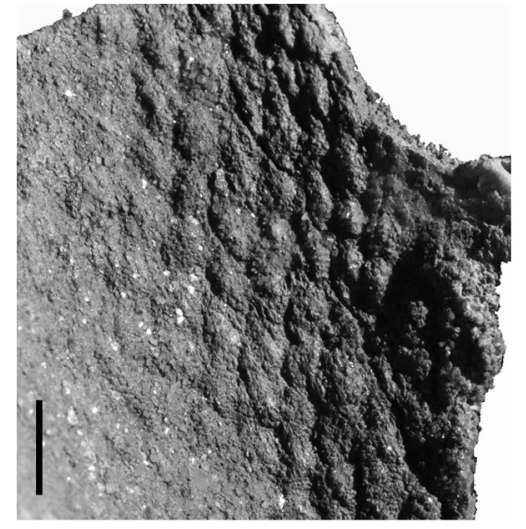

h

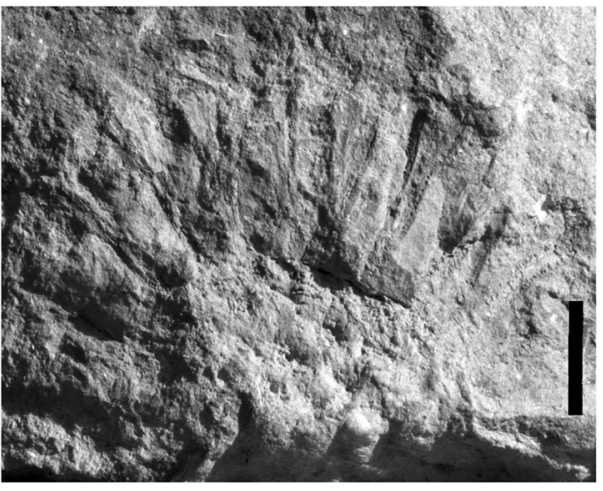

f

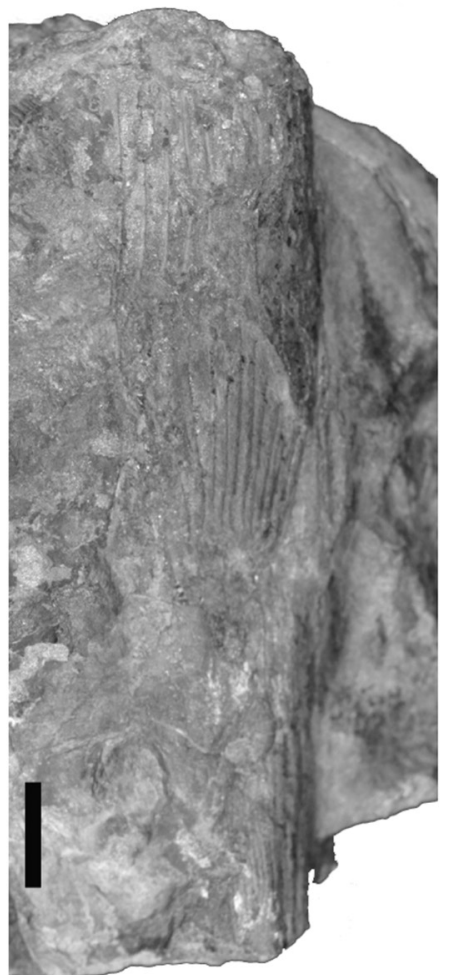

c

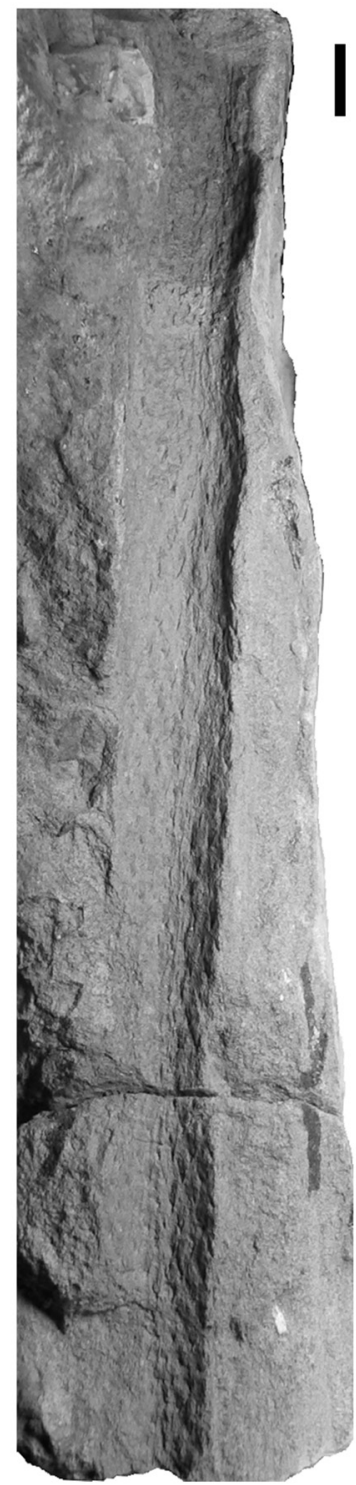

g

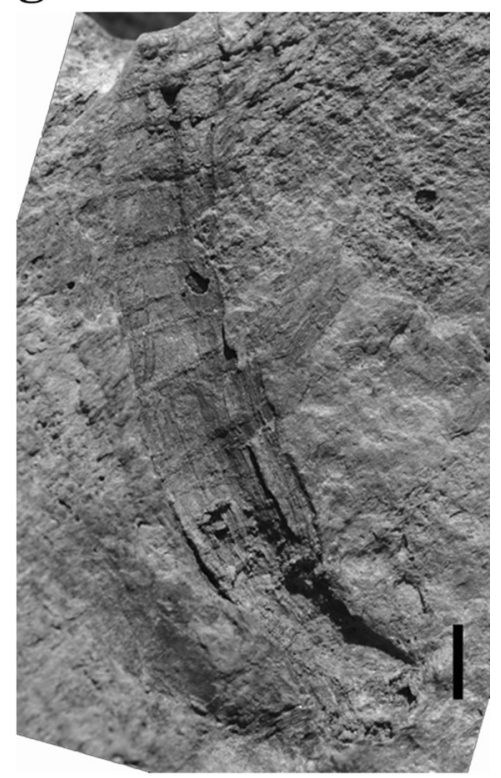




\section{ZONAS CENTROIBÉRICA Y ASTUR-OCCIDENTAL LEONESA: ZAMORA Y LUGO (ESPAÑA)}

En los años setenta del siglo XX aparecen tres trabajos en los que se cita la presencia de plantas vasculares en la provincia de Zamora:

Martínez García (1972) encuentra restos vegetales cerca de la localidad de San Vitero (O de Zamora), en tres lugares dentro del tramo superior de la serie homónima compuesto de alternancias de grauvacas y pelitas cloríticas, pero es, sobre todo, en las zonas más arcillosas de color negro en donde encuentra lo que denomina "tallos y raíces que no han podido ser determinadas". La serie de San Vitero la asigna al Silúrico (Wenlock superior). En el mismo trabajo se cita en la localidad de San Clodio (SE de Lugo) restos de plantas indeterminables que son datadas como silúricas por la similitud estratigráfica, petrográfica y paleobotánica a la serie de San Vitero; previamente, los materiales de San Clodio habían sido asignados con dudas por Riemer (1966: 17) al Carbonífero, resaltando que "La flora está muy mal conservada".

En un trabajo posterior (Martínez García, 1973) vuelve a citar el descubrimiento de fragmentos de plantas en las grauvacas de la serie de San Vitero que sigue considerando del Wenlock superior, aunque como veremos posteriormente Teixeira \& Pais (1973) consideran que estos materiales tendrían una edad comprendida entre el Devónico Superior y el Carbonífero Inferior. En la única figura sobre estos fósiles vegetales que se aporta en la publicación (Martínez García, 1973) se observa una muestra de mano con lo que parece un fragmento de caule y en la leyenda dice haber sido recogida en el $\mathrm{km} \mathrm{27,7}$ de la carretera de Alcañices a Villardeciervos (Zamora).
Parte del material que recogió Martínez García se conserva, en el IMGEMA Jardín Botánico de Córdoba; así, un ápice incompleto y aplastado (Fig. 4.h) determinado por R. H. Wagner como Eoacanthocarpus sp. (Lycopsida, Isoetales), que presenta similitudes con los ejemplares recogidos en el yacimiento de Valdeinfierno (Córdoba) del Tournaisiense superior (ver Wagner, 2001: Fig. 24), y dos muestras de mano con una cicatriz de apéndices de Stigmaria ficoides Sternberg, 1825 (R. H. Wagner, det.) que no aporta gran información. Aunque las Stigmarias (rizomas de Lycophyta) son típicas del Carbonífero, en el Devónico Superior también hay licofitas que deberían tener el mismo sistema radicular.

\section{ZONA CENTROIBÉRICA: BRAGANÇA (PORTU- GAL) Y ZAMORA (ESPAÑA)}

El mismo año de la publicación anterior, Teixeira \& Pais (1973) publican un trabajo en el que citan el descubrimiento de flora encontrada por Teixeira (1970) en Guadramil, en la zona de Bragança (Portugal), y describen la flora encontrada por ellos en Alcañices (Zamora), comparándola con la encontrada por Martínez García (1972, 1973) en San Vitero y San Clodio. La flora encontrada, entre los kilómetros 27 y 28,6 de la carretera de Alcañices a Villardeciervos (Zamora), consiste en restos de caules de cf. Calamites sp. y cf. Sublepidodendron sp. y otros indeterminables. Ambos taxones se figuran en la publicación. Los autores basándose en la estratigrafía y en la flora encontrada asignan las series de ambos lados de la frontera al Devónico Superior o al Carbonífero Inferior, aunque consideran más probable su pertenencia al Devónico. Este material esta desaparecido en la actualidad y puede que se

Figura 2. a, Sphenopteridium keilhaui Nathorst, 1902; Valle de Santullán (Palencia) Fameniense inferior; ejemplar depositado en el IMGEMA Jardín Botánico de Córdoba; escala 3 mm. b, Posible estróbilo de Sphenophyta; Valle de Santullán (Palencia); Fameniense inferior; ejemplar depositado en el IMGEMA Jardín Botánico de Córdoba; escala 3 mm. c, Lepidodendraceae indet. (=Flemingitaceae); Guadalmez (Ciudad Real); Emsiense superior-Givetiense superior; ejemplar depositado en el Museo del Departamento de Geología, Universitat de València; escala $9 \mathrm{~mm}$; fotografía realizada por el Dr. M. Pardo. d, Fragmento de tronco de Lycophyta; Cangas de Onís (Asturias); Fameniense superior-Tournaisiense; ejemplar depositado en el IMGEMA Jardín Botánico de Córdoba; escala 5 mm. e, Fragmento de tronco de Lycophyta; Cangas de Onís (Asturias); Fameniense superior-Tournaisiense; ejemplar depositado en el IMGEMA Jardín Botánico de Córdoba; escala 5 mm. f, Archaeocalamites sp.; Puerto de Guebarnia (Huesca); Devónico; ejemplar depositado en el Museo del Instituto Geológico y Minero de España; escala 10 mm. g, Posible resto vegetal; Gavà (Barcelona); Devónico Inferior; ejemplar depositado en el Museo Geológico del Seminario de Barcelona; escala 4 mm. h, Eoacanthocarpus sp.; San Vitero (Zamora); Devónico Superior o Carbonífero Inferior; ejemplar depositado en el IMGEMA Jardín Botánico de Córdoba; escala 5 mm.

a, Sphenopteridium keilhaui Nathorst, 1902; Valle de Santullán(Palencia); Lower Famennian; specimen lodged in IMGEMA Jardín Botánico de Córdoba; scale bar $3 \mathrm{~mm}$. b. Strobile of probable Sphenophyta; Valle de Santullán(Palencia); Lower Famennian; specimen lodged in IMGEMA Jardín Botánico de Córdoba; scale bar 3mm. c, Lepidodendraceae indet. (=Flemingitaceae); Guadalmez (Ciudad Real); Upper Emsian-Upper Givetian; specimen lodged in Museum of Geology Department, València University; scale bar $9 \mathrm{~mm}$; photographed by Dr. M. Pardo. d, Stem fragment of Lycophyta; Cangas de Onís (Asturias); Upper Famennian-Tournaisian; specimen lodged in IMGEMA Jardín Botánico de Córdoba; scale bar $5 \mathrm{~mm}$. e, Stem fragment of Lycophyta; Cangas de Onís (Asturias);Upper Famennian-Tournaisian; specimen lodged in IMGEMA Jardín Botánico de Córdoba; scale bar $5 \mathrm{~mm}$. f, Archaeocalamites sp.; Guebarnia mountain pass (Huesca); Devonian; specimen lodged in Instituto Geológico y Minero de España Museum; scale bar $10 \mathrm{~mm}$. g, Possible plant remain; Gavà (Barcelona); Lower Devonian; specimen lodged in Seminario de Barcelona Geological Museum; scale bar $4 \mathrm{~mm}$.) h, Eoacanthocarpus sp.; San Vitero (Zamora); Upper Devonian or Lower Carboniferous; specimen lodged in IMGEMA Jardín Botánico de Córdoba; scale bar $5 \mathrm{~mm}$. 
perdiera en 1976, en el incendio de la Facultad de Ciencias de Lisboa (João Pais, 2008, com. escrita).

\section{ZONA CENTROIBÉRICA: BADAJOZ (ESPAÑA)}

Álvarez-Ramis (1981) cita una flora vascular devónica procedente de un yacimiento descubierto por P. Herranz Araujo en la Sierra de Hornachos (SE de Badajoz), datado en el trabajo como Devónico Superior. Más tarde, Herranz Araujo (1985) sitúa geológica y geográficamente el yacimiento y describe los seis niveles de materiales terrígenos en donde encontró los restos de flora. Además se amplía el alcance estratigráfico al Devónico Superior-Carbonífero Inferior (Álvarez Ramis in Herranz Araujo, 1985: 793). El problema es que en el listado de flora que aparece en ambos trabajos (Álvarez-Ramis, 1981; Herranz Araujo, 1985) (ver Tabla 1) se citan taxones típicos del Devónico Inferior (por ejemplo: Zosterophyllum Penhallow, 1892 o Psilophyton Dawson, 1859 aunque este último también se cita, aunque escasamente, en el Devónico Medio), con otros característicos del Devónico Superior- Carbonífero Inferior (como Archaeosigillaria Kidston, 1901, Cyclostigma Haughton, 1859 y Rhacophyton Crépin, 1875, por ejemplo), se cita Archaeopteris, taxón exclusivo del Devónico Superior, y se cita Archaeocalamites del que ya se ha discutido en otro epígrafe su dudosa existencia por debajo del Carbonífero Inferior. Esta mezcla hace que el listado ofrecido resulte inviable para una sola localidad. Otros géneros como Hostimella Stur, 1882 (=Hostinella) y Dawsonites Halle, 1916, son géneros discutidos. Por otro lado, la figuración es de baja calidad por lo que no se puede llegar a ninguna conclusión; incluso algunas de las figuras nos lleva a pensar en simples fragmentos de tallos macerados. Por la figuración, estos restos tan fragmentarios recuerdan a la flora encontrada en Barrancos (Alentejo, Portugal) por Teixeira (1951) y ya comentada en un epígrafe anterior.

Posteriormente, Álvarez-Ramis (1988) citó la especie Sciadophyton steinmanni Kräusel \& Weyland, 1930, sinónimo de Sciadophyton laxum (Dawson, 1871) Steinmann, 1929 (ver Schweitzer, 1980) en una nueva localidad, Monterrubio de la Serena, de la misma provincia (SE de Badajoz) y la dató como Gediniense. La cita corresponde a un Abstract, pero el trabajo sobre este hallazgo nunca se llegó a publicar y, por tanto, el ejemplar no está figurado, lo que hace imposible contrastar la existencia de esta especie en el Devónico español, aunque esta cita fuera recogida por Edwards \& Wellman (2001) en un trabajo de síntesis sobre yacimientos devónicos. Si realmente el material español perteneciera a este género sería un descubrimiento importante que merecería una revisión. Los ejemplares del género Sciadophyton Steinmann, 1929, emend. Kräusel \& Weyland, 1930, son escasos y proceden, en gran parte, de Gaspé, Canadá (Gensel \& Andrews, 1984) o de la localidad tipo, el yacimiento alemán de Wahnbachtal (Kräusel \& Weyland, 1930). Su morfología es característica y pre- senta alrededor de una docena de ejes desnudos que pueden alcanzar hasta más de 10 centímetros de longitud. Los ejes divergen en la base de una "roseta" central y terminan en unas estructuras en forma de copa (ver Kräusel \& Weyland, 1930: fig. 37; Kenrick, 1994: fig. 11).

\section{ZONA CENTROIBÉRICA: CIUDAD REAL (ESPAÑA)}

La última cita de flora vascular devónica que conocemos sitúa el material en un yacimiento cerca de la localidad de Guadalmez (SE de Ciudad Real), con una edad entre el Emsiense superior y el Givetiense superior, alrededor de la laguna estratigráfica del Devónico Medio, en el Miembro Miramonte de la Formación Herrera (Pardo Alonso, 1997). Se trata de un fragmento de molde externo de un pequeño tronco (Fig. 4.c), determinado como Lepidodendraceae indet. (Pardo Alonso, 1997: 132, fig. 10). Esta Familia corresponde a la actual Flemingitaceae (Cleal, 1993) cuyo rango estratigráfico es exclusivamente carbonífero (Tournaisiense superior-Kasimoviense superior) y por tanto este ejemplar deberá ser reestudiado. Fue descubierto en 1991, en areniscas cuarcíticas rojas, muestreando en busca de fauna marina $y$, aunque el ejemplar apareciera en capas de origen marino, lateralmente, en la misma Unidad, existen afloramientos de rocas de origen fluvial (Miguel Pardo, 2007, com. escrita).

En el mismo trabajo se citan "restos vegetales flotados" en areniscas de la parte baja del tramo del Miembro superior de la Formación Herrera, siempre a techo de los estratos. Este material paleobotánico había sido citado en un trabajo anterior (Pardo Alonso \& García-Alcalde, 1996), como "frecuentes restos vegetales flotados" a techo de la Formación Herrera, aunque se aclara más adelante que son "restos vegetales indeterminables". También señalan los autores la aparición de restos vegetales en algunos nódulos de la Formación Guadalmez. En ambos casos, se trata de briznas y restos carbonosos de materia vegetal (Miguel Pardo, 2007, com. escrita). Años antes, se publicó otro trabajo (García-Alcalde et al., 1984), en donde se cita "abundante flora flotada en algunos tramos" al Sur del Río Guadalmez, en capas asignadas por los autores al Devónico Superior (Frasniense alto a Fameniense inferior) en base a la fauna encontrada, que Almela et al. (1962) habían considerado como Viseense superior-Namuriense. Más arriba en la sucesión, en el núcleo del Sinclinal de Guadalmez, vuelven a encontrar flora flotada, en este caso, claramente carbonífera; ya Almela et al. (1962) la habían dado como Westfaliense y los autores, García-Alcalde et al. (1984) la asocian a la flora del yacimiento de Valdeinfierno (Córdoba) de edad Tournaisiense-Viseense Medio (Wagner, 1976). La flora de Guadalmez fue asignada posteriormente al Namuriense inferior (Wagner, 1999).

Hasta la fecha estos son todos los registros de plantas vasculares devónicas en territorio español y siempre tratándose de material flotado en capas marinas. 


\section{EJEMPLARES DE FLORA VASCULAR DEVÓNICA ESPAÑOLA EN INSTITUCIONES ESPAÑOLAS}

Los ejemplares localizados en depósitos de instituciones españolas son muy escasos, y son los siguientes:

\section{1) EL MATERIAL DEPOSITADO EN EL IMGEMA JARDÍN BOTÁNICO DE CÓRDOBA}

Está compuesto por las especies:

* Sphenopteridium keilhauii Nathorst, 1902. Fameniense inferior. Valle de Santullán (loc. 316 de Wagner) (Palencia). Consta de una sóla muestra con un ejemplar fragmentario determinado por H. J. Schweitzer (Fig. 4.a).

* Hyenia sp. Fameniense inferior. Valle de Santullán (loc. 316 de Wagner) (Palencia). Huella y contrahuella, con restos muy fragmentarios, determinadas por R. H. Wagner (Fig. 4.b).

Inicialmente no parece cuestionable la determinación de Sphenopteridium keilhauii Nathorst, 1902, aunque será objeto de un estudio en profundidad.

En cuanto a la asignación del segundo ejemplar al género Hyenia Nathorst, 1914 (Cladoxylales), es discutible ya que, aunque, se asemeja a algunas partes de ejemplares vegetativos de las especies $H$. sphenophylloides $\mathrm{Na}-$ thorst, 1914 y de $H$. ? rhizoides Nathorst,1914, figurados por Nathorst, el parecido es sólo superficial, tanto en el caule como en las hojas.

En el caso de H. sphenophylloides Nathorst, 1914 y de $H$. ? rhizoides Nathorst,1914, se da una costulación longitudinal con un grosor del caule de alrededor de 3-4 mm mientras que en el ejemplar en estudio no se presenta la costulación y el caule tiene un grosor de aproximadamente $1 \mathrm{~mm}$. Las hojas de ambas especies de Hyenia presentan generalmente un ángulo aproximado de $20^{\circ}-30^{\circ}$ con respecto al caule y longitudes de 0,5 a $1,5 \mathrm{~cm}$, bifurcándose en algunos casos. Por el contrario, en el ejemplar estudiado salen perpendiculares al caule y hacia la mitad de su longitud se curvan hacia la parte apical, en un ángulo que varía entre los $70^{\circ}$ y $80^{\circ}$, y los tamaños de hojas son de 1-2 mm. También la especie H. elegans Kräusel \& Weyland, 1926 (Kräusel \& Weyland, 1932), en las partes vegetativas presenta un caule con costulación longitudinal y un grosor de entre 2 y $4 \mathrm{~mm}$, las hojas se bifurcan varias veces y presentan tamaños mayores que en nuestro ejemplar. La estructura de las hojas, en verticilos, se observa bien en la reconstrucción que hace Schweitzer (1990). Queda descartada totalmente la posibilidad de que sea una parte fértil de Hyenia, ya que esta especie presenta las hojas fértiles con una primera división dicotómica y en su parte distal 2 o 3 esporangios de forma elíptica (Fairon-Demaret \& Berry, 2000). Muchos géneros devónicos presentan esporangios que están adheridos a las ramas o se presentan como apéndices que salen de la rama con extensiones distales, dicotomizadas o en racimo.
El ejemplar determinado como Hyenia sp., se asemeja más a un estróbilo que a una parte vegetativa. En concreto, a un estróbilo de Sphenophyta, ya que presenta los esporófilos y láminas distales insertados perpendicularmente al eje. Este ejemplar necesita un estudio en profundidad.

Otros dos ejemplares que se custodian en las colecciones del IMGEMA Jardín Botánico de Córdoba y que provienen de la región de Cangas de Onís (Asturias), que fueron enviados por el Dr. Julivert al Dr. Wagner en 1968 explicando: "hemos encontrado unos vegetales en lo que debe ser la arenisca de La Ermita". Son fragmentos de troncos, atribuibles, con toda certeza a Lycophyta (Figs. 4.d-e), aunque este material no pudo ser determinado en los años 60 por su mala preservación (com. personal Roberto Wagner, 2007). Estos ejemplares fueron recolectados en la Formación Ermita, que está constituida por unas areniscas que fueron consideradas durante mucho tiempo exclusivas del Devónico Superior (Julivert et al., 1968; Truyols Santonja, 1983). En general la Formación Ermita pertenece al Fameniense superior (García-Alcalde, 1995; García-Alcalde et al., 2002), aunque la parte final de la sucesión en algunos lugares puede que sobrepase el Fameniense y llegue al Tournaisiense (García-Alcalde, 1996; García-Alcalde et al., 2002). El análisis del material no ayuda a situarlo en la sucesión, ya que por un lado es fragmentario y está muy mal preservado lo que hace difícil su estudio y, por otro lado, la literatura paleobotánica no refleja cambios entre las Lycophyta del Fameniense superior y las del Tournaisiense inferior, lo que anula, en este caso, la posibilidad de datación. El medio de depósito de la Formación Ermita en Asturias es de plataforma poco a muy poco profunda (García-Alcalde, 1995; García-Alcalde et al., 2002).

Aunque tienen una pésima conservación, con abundantes recristalizaciones de cuarzo en su superficie, en ambas piezas se percibe el dibujo romboidal característico debido a las cicatrices foliares. La diferencia en morfología y dimensiones de esas cicatrices lleva a pensar que podría tratarse de dos especies distintas de licópsidas.

Por último, la colección del IMGEMA Jardín Botánico de Córdoba alberga tres ejemplares colectados cerca de Alcañices (Zamora), uno de ellos determinado como Eoacanthocarpus sp. (R. H. Wagner, det.) (Fig. 4.h). Aunque los registros publicados de este taxón son exclusivamente del Tournaisiense, el alcance estratigráfico total es desconocido, e hipotéticamente podría llegar al Devónico Superior. Los otros dos ejemplares fueron determinados como Stigmaria ficoides Sternberg, 1825 (R. H. Wagner, det.). Por tanto, todo este material podría corresponder al Devónico Superior o al Carbonífero Inferior.

\section{2) MATERIAL DEPOSITADO EN EL MUSEO DEL INSTITUTO GEOLÓGICO Y MINERO DE ESPAÑA}

Se trata de un único ejemplar determinado como $A r$ chaeocalamites sp. (Fig. 4.f), procedente del Puerto de 
Guebarnia (Huesca) y datado como Devónico. Tanto el colector como la persona que hizo la determinación son anónimas. Se determinó en la Escuela Técnica Superior de Ingenieros de Minas de Madrid en 1983 (com. personal Ana Rodrigo, 2005). Se ha comentado anteriormente la problemática de este género en cuanto a su alcance estratigráfico. Aunque no se ha descartado totalmente que pudiera aparecer en el Devónico terminal, es claramente una especie carbonífera y no existe literatura paleobotánica suficiente para contrastar y poder ampliar su alcance estratigráfico al Devónico. La preservación del ejemplar (un contramolde) no es buena, pero la determinación es correcta. Hay que añadir aquí que el punto citado de la provincia de Huesca no ha podido ser encontrado en ningún mapa, y quizás haya existido un error de trascripción en el dato de localidad. Podría tratarse de la localidad de Gavarnie (Francia), en donde sí existe Devónico (Mirouse, 1967, 1992), pero en los trabajos consultados sobre el Devónico del Pirineo español (Waterlot, 1969a, 1969b) no aparece dicha localidad. Por tanto, con este ejemplar se debería ser prudente al asignarlo al Devónico.

\section{3) MATERIAL DEPOSITADO EN EL MUSEO GEO- LÓGICO DEL SEMINARIO DE BARCELONA}

En este Museo está depositado un ejemplar identificado como posible resto vegetal devónico que procede de la localidad de Bruguers, Municipio de Gavà, Barcelona. Fue colectado en capas marinas del Devónico Inferior con abundantes restos de graptolitos, braquiópodos y otra fauna (Racheboeuf et al., 1993; Chlupáč et al., 1997; GutiérrezMarco et al., 1999). El supuesto resto vegetal se asemeja superficialmente a un molde de Archaeocalamites sp., aunque tras un estudio detallado se puede observar la carencia tanto de costillas longitudinales como de las líneas nodales. El ejemplar presenta una estriación longitudinal y pequeñas fracturas rellenadas por sedimento que podrían ser confundidas con los caracteres comentados en un estudio somero (Fig. 4.g).

No es raro el hallazgo de restos vegetales flotados asociados a graptolitos que son los fósiles que datan correctamente las capas. Edwards et al. (2000) citan bastantes ejemplos de estos yacimientos, en donde aparecen taxones como Cooksonia Lang, 1937, Zosterophyllum Penhallow,

\begin{tabular}{|c|c|c|c|c|c|c|c|}
\hline & Especie & Edad & Localidad & $\begin{array}{l}\mathrm{N}^{\mathrm{o}} \text { de } \\
\text { ejemplares }\end{array}$ & Tipo de resto & $\begin{array}{l}\text { Colector/ } \\
\text { Donante }\end{array}$ & $\begin{array}{l}\mathrm{N}^{\circ} \text { de Catálogo/ } \\
\text { Inventario }\end{array}$ \\
\hline \multirow{6}{*}{$\begin{array}{l}\text { IMGEMA. JARDÍN } \\
\text { BOTÁNICO DE } \\
\text { CÓRDOBA }\end{array}$} & Hyenia sp. & Fameniense inf. & $\begin{array}{l}\text { Valle de Santullán } \\
\text { (Palencia) }\end{array}$ & $\begin{array}{l}2 \text { (molde y } \\
\text { contramolde) }\end{array}$ & $\begin{array}{l}\text { Fragmento de } \\
\text { ¿estróbilo? }\end{array}$ & $\begin{array}{l}\text { R.H. Wagner } \\
(1953)\end{array}$ & 49674 GB \\
\hline & $\begin{array}{l}\text { Sphenopteridium } \\
\text { keilhauii Nathorst, } 1902\end{array}$ & Fameniense inf. & $\begin{array}{l}\text { Valle de Santullán } \\
\text { (Palencia) }\end{array}$ & 1 & $\begin{array}{l}\text { Fragmento de } \\
\text { rama }\end{array}$ & $\begin{array}{l}\text { R.H. Wagner } \\
(1953)\end{array}$ & $47830 \mathrm{~GB}$ \\
\hline & ¿Lycopsida? & $\begin{array}{l}\text { Fameniensesup.- } \\
\text { Tournaisiense }\end{array}$ & $\begin{array}{l}\text { Cangas de Onís } \\
\text { (Asturias) }\end{array}$ & 2 & $\begin{array}{l}\text { Fragmento de } \\
\text { tallo }\end{array}$ & $\begin{array}{l}\text { E. Martínez } \\
(1968)\end{array}$ & $10 \mathrm{D}$ \\
\hline & Eoacanthocarpus sp. & $\begin{array}{l}\text { Devónico Sup.- } \\
\text { Carbonífero Inf. }\end{array}$ & San Vitero (Zamora) & $\begin{array}{l}2 \text { (molde y } \\
\text { contramolde) }\end{array}$ & $\begin{array}{l}\text { Ápice } \\
\text { incompleto }\end{array}$ & $\begin{array}{l}\text { E. Martínez } \\
(1972)\end{array}$ & $9 \mathrm{D}$ \\
\hline & $\begin{array}{l}\text { Stigmaria ficoides } \\
\text { Sternberg, } 1825\end{array}$ & $\begin{array}{l}\text { Devónico Sup.- } \\
\text { Carbonífero Inf. }\end{array}$ & San Vitero (Zamora) & 2 & $\begin{array}{l}\text { Cicatrices de } \\
\text { apéndices }\end{array}$ & $\begin{array}{l}\text { E. Martínez } \\
(1972)\end{array}$ & 13D \\
\hline & Resto vegetal & $\begin{array}{l}\text { Devónico Sup.- } \\
\text { Carbonífero Inf. }\end{array}$ & San Vitero (Zamora) & 1 & ¿? & $\begin{array}{l}\text { E. Martínez } \\
(1972)\end{array}$ & $14 \mathrm{D}$ \\
\hline $\begin{array}{l}\text { MUSEO IGME } \\
\text { MADRID }\end{array}$ & Archaeocalamites sp. & ¿Devónico? & $\begin{array}{l}\text { Puerto de } \\
\text { Guebarnia, Huesca }\end{array}$ & 1 & $\begin{array}{l}\text { Fragmento de } \\
\text { tallo. }\end{array}$ & Desconocido & $\begin{array}{l}\text { 1D. Colección } \\
\text { de Invertebrados } \\
\text { y Flora fósil de } \\
\text { España. }\end{array}$ \\
\hline $\begin{array}{l}\text { MUSEO } \\
\text { GEOLÓGICO DEL } \\
\text { SEMINARIO DE } \\
\text { BARCELONA }\end{array}$ & ¿Resto vegetal? & Devónico Inf. & $\begin{array}{l}\text { Bruguers, Municipio } \\
\text { de Gavà, Barcelona. }\end{array}$ & 1 & $\begin{array}{l}\text { Fragmento de } \\
\text { tallo. }\end{array}$ & $\begin{array}{l}\text { Enric Ferrer } \\
(1985)\end{array}$ & $N^{a} 42282$ \\
\hline $\begin{array}{l}\text { MUSEO DEL } \\
\text { DEPARTAMENTO } \\
\text { DE GEOLOGIA, } \\
\text { UNIVERSIDAD } \\
\text { DE VALENCIA }\end{array}$ & $\begin{array}{l}\text { Lepidodendraceae indet. } \\
\text { (=Flemingitaceae) } \\
\text { Restos vegetales } \\
\text { fragmentarios }\end{array}$ & $\begin{array}{l}\text { Emsiense sup. } \\
\text {-Givetiense sup. } \\
\text { Emsiense sup. - } \\
\text { Givetiense sup. }\end{array}$ & $\begin{array}{l}\text { Guadalmez, Ciudad } \\
\text { Real } \\
\text { Guadalmez, Ciudad } \\
\text { Real }\end{array}$ & 8 & $\begin{array}{l}\text { Fragmento de } \\
\text { tallo. } \\
\text { Fragmentos de } \\
\text { tallos }\end{array}$ & $\begin{array}{l}\text { M. V. Pardo } \\
(1991) \\
\text { M. V. Pardo } \\
(1991)\end{array}$ & Sin inventariar \\
\hline
\end{tabular}

Tabla 2. Ejemplares de flora vascular devónica española depositados en museos españoles. Specimens of Devonian vascular plants from Spain lodged in Spanish Museums. 
1892, Drepanophycus Göppert, 1952, en el hemisferio norte o Baragwanathia Lang \& Cookson, 1935, en el hemisferio sur. Sin embargo, sin desechar que pueda ser un resto vegetal, no hemos encontrado caracteres reconocibles para poder asignarlo a un taxón de planta vascular.

\section{4) MATERIAL DEPOSITADO EN EL MUSEO DEL DEPARTAMENTO DE GEOLOGÍA DE LA UNIVER- SITAT DE VALÈNCIA}

Consiste en un molde externo de un tronco en arenisca de cerca de 19 centímetros de longitud y algo menos de un centímetro de anchura (Fig. 4.e), y parte del contramolde de tan sólo 3,5 centímetros, determinado como Lepidodendraceae indet. (=Flemingitaceae) procedente de Guadalmez (Ciudad Real) (Pardo Alonso, 1997). Aunque el material está meteorizado, es reconocible la superficie externa de la corteza que presenta un cierto grado de decorticación, donde se observa el dibujo romboidal formado por los cojinetes foliares. Este ejemplar se encuentra actualmente en estudio para su correcta determinación.

También alberga esta Institución ocho muestras de mano en arenisca, con restos de tallos fragmentarios de la misma localidad.

\section{CONCLUSIONES}

Los restos de plantas vasculares consideradas devónicas que han aparecido en la Península Ibérica desde los primeros descubrimientos de los que se tiene noticia en el siglo XIX, han estado en algún caso mal datados, en algún otro mal determinados y en su mayoría las determinaciones han sido realizadas con precauciones dada la conservación y fragmentación del material. Los pocos fósiles encontrados que corresponden realmente al Devónico son restos flotados que aparecen en yacimientos de borde de cuenca o medios costeros y están asociados a fauna que puede datar las capas, o en formaciones estratigráficas igualmente bien datadas.

En la actualidad los fósiles de plantas vasculares localizados en Instituciones españolas son:

a) Los dos ejemplares que forman parte de la colección del IMGEMA Jardín Botánico de Córdoba y que proceden de Palencia. La determinación de uno de los ejemplares palentinos ha sido cuestionada en este trabajo y está siendo revisada. Así mismo, también se custodian en esta Institución dos ejemplares procedentes de Asturias que pueden pertenecer tanto al Devónico terminal como al Carbonífero basal, dada la información que se sabe de su recolecta y, por último, los ejemplares de Eoacanthocarpus sp. y de Stigmaria ficoides colectados en Zamora que podría pertenecer al Devónico Superior o al Carbonífero Inferior.

b) Un ejemplar de Lepidodendraceae (=Flemingitaceae) y ocho muestras de mano con material fragmentario que están depositados en el Museo del Departamento de Geologia de la Universitat de València y proceden de Ciudad Real. Todo este material está siendo revisado.

c) Un ejemplar de Archaeocalamites perteneciente al Museo del Instituto Geológico y Minero de España que debe ser puesta en duda su asignación estratigráfica ya que existen problemas con el dato de localidad. Además, la presencia de este taxón está cuestionada por debajo del Carbonífero Inferior.

d) Un ejemplar depositado en el Museo Geológico del Seminario de Barcelona que también debe ser puesto en duda ya que no es seguro que se trate de una planta vascular.

e) Los ejemplares portugueses, restos fragmentarios de caules básicamente, se encuentran en el Museu Geológico del Instituto Nacional de Engenharia, Tecnologia e Inovação, y corresponden sólo a las colectas de Nery Delgado (1908) y Perdigão (1964).

Por último, los ejemplares encontrados por Hermite en el siglo XIX en Menorca (casi con toda seguridad carboníferos), el que encontraron Gómez de Llarena en Navarra, Kullmann en Palencia, Teixeira en Bragança y Alentejo, Teixeira y Pais en Zamora y Álvarez Ramis en Badajoz no han podido ser estudiados por estar ilocalizables.

\section{AGRADECIMIENTOS}

Quiero agradecer al Dr. Roberto Wagner (Centro Paleobotánico del Jardín Botánico de Córdoba) sus comentarios y sugerencias sobre diversos aspectos del trabajo que han conseguido mejorarlo considerablemente, su ayuda con la bibliografía y el facilitarme los detalles sobre el origen de los ejemplares devónicos que se conservan en el IMGEMA Jardín Botánico de Córdoba. También agradezco al Dr. Sebastián Calzada (Museo Geológico del Seminario de Barcelona), al Dr. Miguel Pardo (Universitat de València), a la Dra. Ana Rodrigo (Museo Geominero) y al Dr. Miguel Ramalho (Museu Geológico del Instituto Nacional de Engenharia, Tecnologia e Inovação) el haberme facilitado la información del material devónico que custodian, y al Dr. João Pais (Instituto Nacional de Engenharia, Tecnologia e Inovação) la información sobre los ejemplares colectados por él en Bragança y Zamora. Por último, quiero también agradecer las sugerencias de la Dra. Carmen Diéguez (Museo Nacional de Ciencias Naturales), los comentarios al texto de un revisor anónimo, el trabajo de los editores Dr. Miguel Pardo y Dr. Rodolfo Gozalo (Universitat de València) y la colaboración de la Dra. Carmen Álvarez-Vázquez (IMGEMA Jardín Botánico de Córdoba), del Dr. José Luis Ubera (Universidad de Córdoba) y de D. Pedro Berjillos (Universidad de Córdoba).

\section{BIBLIOGRAFÍA}

Almela, A., Alvarado, M., Coma, J., Felgueroso, C. \& Quintero, I. 1962. Estudio Geológico de la región de Almadén. Boletín Instituto Geológico y Minero de España, 73, 195-327. Álvarez-Ramis, C. 1981. Devonian plants from Hornachos (Badajoz), Spain. The Palaeobotanist, 28-29, 15-19. 
Álvarez-Ramis, C. 1988. Presence de Sciadophyton steinmanni Carrusel \& Weyland dans le Gedinien de la Serie Devonienne de Monterrubio de la Serena (Badajoz, Espagne). Seminaire de Paleobotanique. Resumes. Organisation Francaise de Paleobotanique, Lille, France, 1.

Banks, H. P. 1981. Time of appearance of some plant biocharacter during Siluro-Devonian Time. Canadian Journal of Botany, 59, 1292-1296.

Berry, C. M. 2005. "Hyenia" vogtii Høeg from the Middle Devonian of Spitsbergen- Its morphology and systematic position. Review of Palaeobotany and Palynology, 135, 109-116.

Berry, C. M. \& Fairon-Demaret, M. 1997. A Reinvestigation of the Cladoxylopsid Pseudosporochnus nodosus Leclercq Et Banks from the Middle Devonian of Goe, Belgium. International Journal of Plant Sciences, 158, 350-372.

Berry, C. M. \& Fairon-Demaret, M. 2001. The Middle Flora Revisited. In: Plants Invade the Land. Evolutionary \& Environmental Perspective (Eds. P.G. Gensel \& D. Edwards). Columbia University Press, New York, 120-139.

Berry, C. M. \& Wang, Y. 2006. A new plant attributed to Cladoxylopsida from the Middle Devonian of Yunan Province, China. Review of Palaeobotany and Palynology, 142, 63-78.

Boureau, E. 1964. Traité de Paléobotanique. T. III. Spheno-

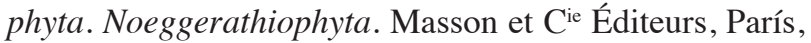
$1-544$.

Bourrouilh, R. 1970-1972. Mapa Geológico de España. Menorca. E: 1:200.000. Primera edición. Instituto Geológico y Minero de España, 1-50.

Brauer, D.F. 1981. Heterosporous, Barinophytacean plants from the Upper Devonian of North America and discussion of the possible affinities of the Barinophytaceae. Review of Palaeobotany and Palynology, 33, 347-362.

Cleal, C.J. 1993. Pteridophyta. In: The Fossil Record 2 (Ed. M.J. Benton). Chapman \& Hall, Londres, 779-794.

Cleal, C. J. \& Thomas, B. A.1995. Palaeozoic Palaeobotany of Great Britain. Geological Conservation Review Series, Chapman \& Hall, London, 1-295.

Chlupáč, I., Ferrer, E., Magrans, J., Mañé, R. \& Sanz, J. 1997. Early Devonian eurypterids with Bohemian affinities from Catalonia (NE Spain). Batalleria, 7, 9-21.

Cressler, W. L. \& Pfefferkorn, H. W. 2005. A Late Devonian Isoetalean Lycopsid, Otzinachsonia beerboweri, gen. et. sp. nov., from North-Central Pennsylvania, USA. American Journal of Botany, 92, 1131-1140.

Edwards, D. 2006. Danziella artesiana, a new name for Zosterophyllum artesianum from the Lower Devonian of Artois, northern France. Review of Palaeobotany and Palynology, 142, 93-101.

Edwards, D., Kerp, H. \& Hass, H. 1998. Stomata in early land plants: an anatomical and ecophysiological approach. Journal of Experimental Botany, 49, 255-278.

Edwards, D. \& Wellman, Ch. 2001. Embryophytes on Land: The Ordovician to Lochkovian (Lower Devonian) Record. In: Plants Invade the Land. Evolutionary \& Environmental Perspective (Eds. P.G. Gensel \& D. Edwards). Columbia University Press, New York, 3-28.
Edwards, D., Fairon-Demaret, M. \& Berry, C. M. 2000. Plant megafossils in Devonian stratigraphy: a progress report. Courier Forschunginstitut Seckenberg, 220, 25-37.

Edwards, D., Morel, E. Poiré, D. G. \& Cingolani, C. A. 2001. Land plants in the Devonian Villavicencio Formation, Mendoza Province, Argentina. Review of Palaeobotany and Palynology, 116, 1-18.

Fairon-Demaret, M. 1979. Estinnophyton wahnbachense (Kräusel \& Weyland) comb. nov. une plant remarkable du Siegenian d'Allemagne. Review Palaeobotany and Palynology, 28, 145-160.

Fairon-Demaret, M. \& Berry, C. M. 2000. A reconsideration of Hyenia elegans Krausel et Weyland and Hyenia "complexa" Leclercq: two Middle Devonian Cladoxylopsids from Western Europe. International Journal of Plant Sciences, 161, 473-494.

García-Alcalde, J. L. 1995. L'évolution paléogéographique pré-varisque de la Zone Cantabrique septentrionale (Espagne). Revista Española de Paleontología, 10, 9-29.

García-Alcalde, J. L. 1996. El Devónico del Dominio AsturLeonés en la zona cantábrica ( $\mathrm{N}$ de España). Revista Española de Paleontología, $\mathbf{N}^{\mathbf{0}}$ Extraordinario, 58-71.

García-Alcalde, J. L., Arbizu, M. A., Pardo Alonso, M. V. \& García-López, S. 1984. El Límite Devónico-Carbonífero en el área de Guadalmez-Santa Eufemia (Provs. De Ciudad Real y Córdoba, Sierra Morena, España). I Congreso Español de Geología, 1, 421-430.

García-Alcalde, J., Montesinos, J. R., Truyóls-Massoni, M., García-López, S., Arbizu, M. A. \& Soto, F. 1988. El Silúrico y el Devónico del Dominio Palentino (NO de España). Revista de la Sociedad Geológica de España, 1, 7-13.

García-Alcalde, J., Carls, P., Pardo Alonso, M. V., Sanz López, J., Soto, F., Truyols-Massoni, M. \& Valenzuela-Ríos, J. I. 2002. Devonian. In: The Geology of Spain (Eds. W. Gibbons \& T. Moreno).The Geological Society, London, 67-91.

Gensel, P. G. \& Albright, V. M. 2006. Leclercqia complexa from the Early Devonian (Emsian) of northern New Brunswick, Canada. Review of Palaeobotany and Palynology, 142, 103-121.

Gensel, P. G. \& Andrews, H. N. 1984. Plant Life in the Devonian. Praeger, New York, 1-380.

Gensel, P. G. \& Kasper, A. E. 2005. A new species of the Devonian lycopod genus, Leclercqia, from the Emsian of New Brunswick, Canada. Review of Palaeobotany and Palynology, 137, 105-123.

Gerrienne, P., Bergamaschi, S., Pereira, E., Rodrigues, M-A. C. \& Steemans, P. 2001. An Early Devonian flora, incluiding Cooksonia, from the Paraná Basin (Brazil). Review of Palaeobotany and Palynology, 116, 19-38.

Gerrienne, P., Dilcher, D. L., Bergamaschi, S., Milagres, I., Pereira, E. \& Rodrigues, M-A. C. 2006. An exceptional specimen of the early land plant Cooksonia paranensis, and a hypothesis on the life cycle of the earliest eutracheophutes. Review of Palaeobotany and Palynology, 142, 123-130.

Gómez de Llarena, J. 1950. Breve noticia sobre hallazgos paleontológicos en el Paleozoico de los Pirineos Vascos. Estudios Geológicos, 11, 245-247. 
Gómez de Llarena, J. 1956. Datos paleontológicos sobre los terrenos paleozoicos del valle alto del Arga, Quinto Real (Navarra) e Irún (Guipuzcoa). Extrait des Actes du II Congrés International D’Études Pyrénees. Luchon-Pau, 1954, T.2, Sect.1. Toulouse, 61-71.

Göppert, H. R. 1847. Über die fossile Flora der Grauwacke oder des Übergangs-Gebirges, besonders in Schlesien. Neues Jahrbuch für Mineralogie, Geologie und Paläontologie, 675-686.

Göppert, H. R. 1852. Fossile Flora des Ubergangsgebirges. Verhandlungen der Kaiserlichen Leopoldinisch-Carolinischen Akademie der Naturforscher. Breslau und Bonn. 1-299.

Gutiérrez-Marco, J.C., Ferrer, E., Robardet, M. \& Roqué Bernal, J. 1999. Graptolitos multiramosos del Devónico de las Cadenas Costeras Catalanas (noreste de España). Temas Geológico-Mineros ITGE, 26, 610-617.

Hammond, S. E. \& Berry, C. 2005. A new species of Tetraxylopteris (Aneurophytales) from the Devonian of Venezuela. Botanical Journal of the Linnean Society, 148, 275-303.

Hao, S-G., Wang, D-M. \& Wang, Q. 2004. A new species of Estinnophyton from the Lower Devonian Posongchong Formation, Yunnan, China; its phylogenetic and palaeophytogeographical significance. Botanical Journal of the Linnean Society, 146, 201-216.

Hermite, H. 1879. Etudes géologiques des Iles Baleares. 1 ère partie: Majorque et Minorque. F. Savy, Paris, 1-357. [Traduc.: Estudio Geológico de las Islas Baleares. Mallorca y Menorca. Boletín Comisión del Mapa Geológico de España (año 1888), 15, 2-241].

Hartman, C. M. \& Banks H. P. 1980. Pitting in Psilophyton Dawsonii, an Early Devonian Trimerophyte. American Journal of Botany, 67, 400-412.

Herranz Araujo, P. 1985. El Precámbrico y su cobertera paleozoica en la región centro-oriental de la provincia de Badajoz (II). Seminarios de Estratigrafía, Serie Monografías, 10, 1-1.342.

Höeg, O. A. 1967. Psilophyta. In: Traité de Paléobotanique (Ed. E. Boureau) Tomo II Bryophyta, Psilophyta, Lycophyta. Masson et $C^{\text {ie }}$ Editeurs, 191-433.

Jarvis, D. E. 2000. Palaeoenvironment of the plant bearing horizons of the Devonian-Carboniferous Kiltorcan Formation, Kiltorcan Hills, Co. Kilkenny, Ireland. In: New Perspectives on the Old Red Sandstone (Eds. P. F. Friend \& B. P. J. Williams). Geological Society, Special Publications, 180, London, 333-341.

Julivert, M., Marcos, A., Philippot, A. \& Henry, J. L. 1968. Sobre la extensión de las pizarras ordovícicas al E de la Cuenca Carbonífera Central de Asturias. Breviora Geologica Asturica, 4, 1-4.

Kenrick, P. 1994. Alternation of generations in land plants: new phylogenetic and palaeobotanical evidence. Biological Review, 69, 293-230.

Kerp, H., Hass, H. \& Mosbrugger, V. 2001. New Data on Nothia aphylla Lyon 1964 ex-El-Saadawy et Lacey 1979, a poorly known plant from the Lower Devonian Rhynie Chert. In: Critical Moments \& Perspectives in
Earth History and Paleobiology Series. Plants invade the Land. Evolutionary and Environmental Perspectives (Eds. Gensel \& Edwards). Columbia University Press, 52-82.

Kullmann, J. 1960. Die Ammonoidea des Devon im Kantabrischen Gebirge (Nordspanien). Akademie der Wissenschaften und der Literatur. Abhandlungen der MathematischNaturwissenschaftlichen Klasse, 1960 (7), 1-105.

Kräusel, R. \& Weyland, H. 1930. Die flora des deutschen Unterdevons. Abhandlungen der Preussischen Geologischen Landesanstalt, Neue Folge, 131, 1-92.

Kräusel, R. \& Weyland, H. 1932. Pflanzenreste aus dem Devon. III. Über Hyenia Nath. Senckenbergiana, 14, 274-280.

Mallada, L. 1892. Catálogo General de las Especies Fósiles encontradas en España. Boletín de la Comisión Mapa Geológico de España, 18, 1-254. [Ed. Facsímil: Librería París-Valencia. Valencia (año 1991), 1-253].

Mallada, L. 1898. Explicación del Mapa Geológico de España. Tomo III. Sistemas Devoniano y Carbonífero. Memorias de la Comisión del Mapa Geológico de España, 21, 1-405. [Segunda edición, año 1927, 1-415].

Mamay, S. H. \& Bateman, R. M. 1991. Archaeocalamites lazarii, sp. nov.: The range of Archaeocalamitaceae extended from the Lowermost Pennsylvanian to Mid-Lower Permian. American Journal of Botany, 78, 489-496.

Martínez García, E. 1972. El Silúrico de San Vitero (Zamora). Comparación con series vecinas e importancia orogénica. Acta Geológica Hispánica, 7, 104-108.

Martínez García, E. 1973. Deformación y metamorfismo en la zona de Sanabria. Studia Geológica, 5, 7-106.

Meyer-Berthaud, B. \& Gerriene, P. 2001. Aarabia, a new Early Devonian vascular plant from Africa (Morocco). Review of Palaeobotanica and Palynology, 116, 39-53.

Mirouse, R. 1967. Le Devonien des Pyrenees Occidentales et Centrales (France). In: International Symposium on the Devonian System (Ed. D.H. Oswald). Alberta Society of Petroleum Geologists, Calgary, vol. I, 153-170.

Mirouse, R. 1992. Pyrénées Centrales Franco-Espagnoles. Guides Géologiques Régionaux. Masson, Paris, 1-176.

Nathorst, A. G. 1914-15. Zur Devonflora des westlichen Norwegens. Bergens Museums Aarbok, 9, 1-34.

Nery Delgado, J. F. 1908. Système Silurique du Portugal. Memories e Comunicações dos Serviços Geológicos de Portugal, 1-245.

Oliveira, J. T., Pereira, Z., Fernandes, P. \& Matos, J. 2007. Palynostratigraphy of the Lower Devonian Sucession, Barrancos Region, Ossa Morena Zone. In: Palynostratigraphic contributions to the understanding Ossa Morena and South Portuguese Zone Geology, Portugal. Cimp Lisbon'07. Joint Meeting of Spores/Pollen and Acritarch Subcommissions, 13-17.

Pardo Alonso, M. V. 1997. El Devónico Meridional de la Zona Centroibérica. Tesis Doctoral, Universidad de Oviedo, 1-472 (inédito).

Pardo Alonso, M. V. \& García-Alcalde, J. L. 1996. El Devónico de la Zona Centroibérica. Revista Española de Paleontología, $\mathbf{N}^{\circ}$ Extraordinario, 72-81.

Pereira, Z, Piçarra, J. M. \& Oliveira, J. T. 1999. Lower De- 
vonian palynomorphs from the Barrancos region, Ossa Morena Zone, Portugal. Bollettino della Società Paleontologica Italiana, 38, 239-245.

Racheboeuf, P.R., Ferrer Batet, E. \& Magrans, J. 1993. Un nouvel assemblage faunique du Dévonien inférieur de Catalogne (NE de l'Espagne). Treballs del Museu de Geologia de Barcelona, 3, 5-18.

Remy, W., Taylor, T.N., Hass, H. \& Kerp, H. 1994. Four Hundred-Million-Year-Old Vesicular Arbuscular Mycorrhizae. Proceedings of the National Academy of Sciences of the United States of America, 91, 11841-11843.

Riemer, W. 1966. Datos para el conocimiento de la estratigrafía de Galicia. Notas y Comunicaciones del Instituto Geológico y Minero de España, 81, 7-20.

Roth-Nebelsick, G., Grimm, G., Mosbrugger, V., Hass, H. \& Kerp, H. 2000. Morphometric analysis of Rhynia and Asteroxylon: testing functional aspects of early land evolution. Paleobiology, 26, 405-418.

Schweitzer, H-J. 1980. Die gattungen Taeniocrada White und Sciadophyton Steinmann im Unterdevon des Rheinlandes. Bonner Paläeobotanische Mitteilungen, 5, 1-38.

Schweitzer, H-J. 1990. Pflanzen erobern das land. Kleine Senckenberg-Rheine, 18, 1-75.

Soria, A. \& Meyer-Berthaud, B. 2004. Tree fern growth strategy in the Late Devonian Cladoxylopsid species Pietzschia levis from the study of its stem and root system. American Journal of Botany, 91, 10-23.

Stein, W. E., Mennolini, F., van Aller Hernick, L. Landing, E. \& Berry, C. M. 2007. Giant cladoxylopsid trees resolve the enigma of the Earth's earliest forest stumps at Gilboa. Nature, 446 (5705), 904-907.

Stockmans , F. 1940. Végétaux du Dévonien Supérieur de la Belgique. Mémoires du Musée Royal d'Histoire Naturelle de Belgique, 110, 1-85.

Stur, D. 1875. Die Culm-Flora des Mährisch-Schlesischen Dachschiefers. Abhandlungen der K. K. geologischen Reichsanstalt, Band 8, 1-106.

Taylor, T. N. \& Krings, M. 2005. Fossil microorganisms and land plants: Associations and interactions. SYMBIOSIS, 40, 119-135.

Taylor, T. N., Kerp, H. \& Hass, H. 2005. Life history biology of early land plants: Deciphering the gametophyte phase. PNAS, 12, 5892-5897.

Taylor, T. N., Krings, M. \& Kerp, H.. 2006. Hasiella monospora gen. et sp. nov., a microfungus from the 400 million years old Rhynie chert. Mycological Research, 110, 628-632.

Teixeira, C. 1951. Notas sobre a geologia da Região de Barrancos e, em especial, sobre a sua flora de Psilofitíneas. Сотиnicações dos Serviços Geológicos de Portugal, 32, 5-13.

Teixeira, C. 1970. Restos de vegetais em ftanitos silúricos de Guadramil. Naturália, 10 (2), 5.

Teixeira, C. 1982a. Contribuições para o conhecimento geológico da região de Barrancos (Alentejo, Portugal). 1. Nouvelles Données Paléontologiques et Stratigraphiques sur le Paléozoïque de la région entre Barrancos et Serpa, au Portugal. $2^{a}$ Réunion sur la Géologie de la zona OssaMorena. Lisboa, Portugal, 1-7.
Teixeira, C. 1982b. O Eodevónico da região entre a fronteira de Barrancos e a vila de Serpa. Memórias da Academia das Ciências de Lisboa, 24, 105-125.

Teixeira, C. \& Pais, J. 1973. Sobre a presença de Devonico na região de Bragança (Guadramil e Mofreita) e de Alcañices (Zamora). Boletim da Sociedade Geológica de Portugal, 18, 199-202.

Teixeira, C. \& Thadeu, D. 1967. Le Devonian du Portugal. In: International Symposium on the Devonian System (Ed. D.H. Oswald). Alberta Society of Petroleum Geologists, Calgary, Vol. I, 189-199.

Truyols Santonja, J. 1983. El Carbonífero Inferior y Medio de la Región del Ponga. In: X Congreso Internacional de Estratigrafía y Geología del Carbonífero: Carbonífero y Pérmico de España (Coord. Carlos Martínez Díaz). Instituto Geológico y Minero de España, 82-94.

Wagner, R. H. 1966. Notes on the Geology of Palaeozoic rocks in the Northeastern part of the province of Palencia, N. W. Spain. Notas y Comunicaciones Instituto Geológico y Minero de España, 86, 31-40.

Wagner, R. H. 1971. Carboniferous Nappe Structures in North-Eastern Palencia (Spain). Trabajos de Geología, 4, 431-459.

Wagner, R. H. 1976. The Valdeinfierno sequence (Prov. Córdoba): its tectonic, sedimentary and floral significance. Annales de la Société Géologique du Nord, 98, 59-66.

Wagner, R. H. 1984. Megafloral zones of the Carboniferous. Compte Rendu Neuvième Congrès International de Stratigraphie et de Géologie du Carbonifère. Washington and Champaign-Urbana, 1979, 2, 109-134.

Wagner, R. H. 1999. Macrofloras del Carbonífero/Pérmico. In: La Huella del Pasado. Fósiles de Castilla-La Mancha (Coord. E. Aguirre \& I. Rábano). Patrimonio Histórico. Arqueología. Castilla-La Mancha, 77-98.

Wagner, R. H. 2001. Fósiles vegetales. Serie Recursos Naturales de Córdoba ${ }^{\circ}$ 6, Diputación de Córdoba, 1-105.

Wagner, R. H., Carballeira, J., Ambrose, T. \& Martínez García, E. 1984. Memoria Hoja 107. Barruelo de Santullán. Mapa Geológico de España. IGME, 1-113.

Wang, Y. \& Berry, C. 2006. Morphology of a non-pseudosporochnalean cladoxylopsid from the Middle Devonian of Yunnan, South China. Palaeoworld, 15, 54-67.

Wang, Y. \& Xu, H. H. 2005. Sublepidodendron grabaui comb. nov., a lycopsid from the Upper Devonian of China. Botanical Journal of the Linnean Society, 149, 299-311.

Wang, D-M, Hao, S-G \& Wang, Q. 2005a. Rotafolia songziensis gen. et comb. nov., a sphenoid from the Late Devonian of Hubei, China. Botanical Journal of the Linnean Society, 148, 21-37.

Wang, Q., Geng, B-Y \& Dilcher, D.L. 2005b. New perspective on the architecture of the Late Devonian arborescent lycopsid Leptophloeum rhombicum (Leptophloeaceae). American Journal of Botany, 92, 83-91.

Waterlot, M. 1969a. Contribution à l'étude géologique du Carbonifère anté-Stéphanien des Pyrénées Centrales Espagnoles. Memorias del Instituto Geológico y Minero de España, 70, 1-259. 
Waterlot, M. 1969b. Grands faits stratigraphiques et paléogéographiques du Carbonifère anté-stéphanien des Pyrénées centrales espagnoles. Bulletin Société Géologique de France, $7^{\text {eme }}$ ser. 11, 502-510.

White, D. 1903. Description of fossil algae from the Chemung of New York with remarks on the genus Haliserites Sternberg. New York State Museum Annals, 55, 593-605.
Xu, H-H \& Berry, C. 2008. The Middle Devonian lycopsid Haskinsia Grierson et Banks from the Ruppert Coast, Marie Byrd Land, West Antarctica. Review of Palaeobotany and Palynology, (in press).

Manuscrito recibido: 3 de Diciembre, 2007 Manuscrito aceptado: 28 de Julio, 2008 


\section{REVISTA ESPAÑOLA DE PALEONTOLOGÍA}

\section{NÚMERO EXTRAORDINARIO X}

(XIX Jornadas de Paleontología, Morella, 16-18 de octubre de 2003)

J. V. SANTAFÉ LLOPIS. Presentación

J. L. SANZ. Aproximación histórica al género Iguanodon. [Historical approach at genus Iguanodon.]

X. PEREDA SUBERBIOLA \& J.I. RUIZ-OMEÑACA. Los primeros descubrimientos de dinosaurios en España. [The first dinosaur discoveries in Spain.]

J.M. GASULLA ASENSIO. Los dinosaurios de Morella (Castellón, España): historia de su investigacion. [The dinosaurs of Morella (Castellón, Spain): History of their investigation.]

O. FERRER \& J. M. DE GIBERT. Presencia de Teredolites en la Formación Arcillas de Morella (Cretácico Inferior, Castellón). [Presence of Teredolites in the Morella Mudstone Formation (Lower Cretaceous, Castellón).]

A.M. BRAVO, B. VILA, A. GALOBART \& O. OMS. Restos de huevos de dinosaurio en el Cretácico Superior del sinclina de Vallcebre (Berguedà, provincia de Barcelona). [Dinosaur egg remains from the Upper Cretaceous of Vallcebre Syncline (Berguedà, Barcelona Province).]

J.L. BARCO, J. I. CANUDO, J. I. RUIZ-OMEÑACA \& J.L. RUBIO. Evidencia icnológica de un dinosaurio terópodo gigante en el Berriasiense (Cretácico Inferior) de Laurasia (Las Villasecas, Soria, España). [Ichnological evidence of a giant theropod dinosaur in Berriasian (Lower Cretaceous) of Laurasia (Las Villasecas, Soria, Spain).]

F. TORCIDA FERNÁNDEZ-BALDOR, J.I. RUIZ-OMEÑACA, L.A. IZQUIERDO MONTERO, D. MONTERO HUERTA, G. PÉREZ MARTÍNEZ, P. HUERTA HURTADO \& V. URIÉN MONTERO. Dientes de un enigmático dinosaurio ornitópodos en el Cretácico Inferior de Burgos (España). [Enigmatic ornithopod teeth from the Lower Cretaceous of Burgos (Spain).]

C. FUENTES VIDARTE, M. MEIJIDE CALVO, F. MEIJIDE FUENTES \& M. MEIJIDE FUENTES. Fauna de vertebrados del Cretácico Inferior del Yacimiento de "Zorralbo" en Golmayo (Soria, España). [Vertebrate fauna (Lower Cretaceous) of the site "Zorralbo" a Golmayo (Soria, Spain).]

M. SUÑER, C. DE SANTISTEBAN \& A. GALOBART. Nuevos restos de Theropoda del Jurásico Superior - Cretácico Inferior de la Comarca de los Serranos (Valencia). [ New Upper Jurassic - Lower Cretaceous Theropoda remains from 'Los Serranos' region (Valencia).]

F.J. RUIZ-SÁNCHEZ, C. DE SANTISTEBAN \& J.I. LACOMBA. Cazuma 1, nueva localidad de micromamíferos (Mammalia, Rodentia) del Aragoniense Superior en la cuenca de Quesa - Bicrop (prov. Valencia). [Cazuma 1, new micromammal locality (Mammalia, Rodentia) of Late Aragonian of the Quesa - Bicorp basin (Valencia, Spain).]

M.A. SALINAS JAQUES. Las hipótesis de Eduardo Boscá sobre la alimentación del megaterio y el montaje del ejemplar de la Colección Rodrigo Botet (1902-1928). [The Eduardo Bosca's hypothesis about the megatherium's feeding and the assembly of the Rodrigo Botet Collection's specimen (1902-1928).]

F.J. RUIZ-SÁNCHEZ. La legislación de medio ambiente y la protección del patrimonio paleontológico en la Comunidad Valenciana (España). [The environmental legislation and paleontological heritage protection in the Valencian Community (Spain).] 